JOURNAL OF THE

AMERICAN MATHEMATICAL SOCIETY

Volume 16, Number 4, Pages 751-777

S 0894-0347(03)00427-2

Article electronically published on March 21, 2003

\title{
QUASIANALYTIC DENJOY-CARLEMAN CLASSES AND O-MINIMALITY
}

\author{
J.-P. ROLIN, P. SPEISSEGGER, AND A. J. WILKIE
}

\section{INTRODUCTION}

The work in this paper has been motivated by two questions from the theory of o-minimality (see for instance [6]): (1) Does every o-minimal expansion of the real field admit analytic cell decomposition? (2) Does there exist a "largest" o-minimal expansion $\mathcal{M}$ of the real field, in the sense that any other o-minimal expansion of the real field is a reduct of $\mathcal{M}$ ? We describe here a new method of constructing o-minimal structures, based on a normalization algorithm inspired by Bierstone and Milman [4. We then apply this construction to certain quasianalytic DenjoyCarleman classes (already suggested by Van den Dries in [6]) and thereby answer both questions negatively.

Let $M=\left(M_{0}, M_{1}, \ldots\right)$ with $1 \leq M_{0} \leq M_{1} \leq \cdots$ be a sequence of real numbers and $B=\left[a_{1}, b_{1}\right] \times \cdots \times\left[a_{n}, b_{n}\right]$ with $a_{i}<b_{i}$ for $i=1, \ldots, n$. We let $\mathcal{C}_{B}^{0}(M)$ be the collection of all functions $f: B \longrightarrow \mathbb{R}$ for which there exist an open neighborhood $U$ of $B$, a $C^{\infty}$ function $g: U \longrightarrow \mathbb{R}$ and a constant $A>0$ (all depending on $f$ ) such that $f=g \mid B$ and

$$
\left|g^{(\alpha)}(x)\right| \leq A^{|\alpha|+1} \cdot M_{|\alpha|} \quad \text { for all } x \in U \text { and } \alpha \in \mathbb{N}^{n},
$$

where $|\alpha|:=\alpha_{1}+\cdots+\alpha_{n}$. We call $\mathcal{C}_{B}^{0}(M)$ the Denjoy-Carleman class on $B$ associated to $M$. (If $M_{i}=i$ ! for all $i \geq 0$, then $\mathcal{C}_{B}^{0}(M)$ is the class of all real-valued functions on $B$ that extend analytically to an open neighborhood of $B$.) Without loss of generality (see [11, 19]), we shall assume that $M$ is logarithmically convex (or log-convex for short), that is, $M_{i}^{2} \leq M_{i-1} M_{i+1}$ for all $i>0$.

The class $\mathcal{C}_{B}^{0}(M)$ is called quasianalytic if for any $f \in \mathcal{C}_{B}^{0}(M)$ and any $x \in B$, the Taylor series $\widehat{f}_{x}$ of $f$ at $x$ uniquely determines $f$ among all functions in $\mathcal{C}_{B}^{0}(M)$. It is well known [11, 19] that $\mathcal{C}_{B}^{0}(M)$ is quasianalytic if and only if

$$
\sum_{i=0}^{\infty} \frac{M_{i}}{M_{i+1}}=\infty
$$

In general, the classes $\mathcal{C}_{B}^{0}(M)$ will not be closed under differentiation. However, the classes $\mathcal{C}_{B}(M):=\bigcup_{j=0}^{\infty} \mathcal{C}_{B}^{0}\left(M^{(j)}\right)$, where $M^{(j)}:=\left(M_{j}, M_{j+1}, \ldots\right)$, obviously are. Moreover, we would like the system of all $\mathcal{C}_{B}(M)$, where $M$ is fixed and

Received by the editors February 19, 2001.

2000 Mathematics Subject Classification. Primary 14P15, 03C64; Secondary 32S45.

Key words and phrases. Quasianalytic classes, o-minimal structures, resolution of singularities. Supported in part by CNRS, NSERC grant OGP0009070 and NSF grant DMS-9988453. 
$B=\left[a_{1}, b_{1}\right] \times \cdots \times\left[a_{n}, b_{n}\right]$ with $a_{i}<b_{i}$ for $i=1, \ldots, n$ and $n \in \mathbb{N}$, to be closed under compositions, taking implicit functions and division by monomial terms. We show in the appendix, based on the existing literature, that this is the case if the sequence $M$ is strongly log-convex, which means by definition that the sequence $\left(M_{i} / i !\right)$ is log-convex.

For each $n \in \mathbb{N}$ and $f \in \mathcal{C}_{[-1,1]^{n}}(M)$, we define $\widetilde{f}: \mathbb{R}^{n} \longrightarrow \mathbb{R}$ by $\widetilde{f}(x):=f(x)$ if $x \in[-1,1]^{n}$ and $\widetilde{f}(x):=0$ otherwise. We let $\mathbb{R}_{\mathcal{C}(M)}$ be the expansion of the real field by all $\tilde{f}$ for $f \in \mathcal{C}_{[-1,1]^{n}}$ and $n \in \mathbb{N}$. Building on the closure properties mentioned above, we establish

Theorem 1. If $M$ is strongly log-convex and satisfies (QA), then the structure $\mathbb{R}_{\mathcal{C}(M)}$ is model complete, o-minimal, polynomially bounded and admits $C^{\infty}$ cell decomposition.

The model-completeness of $\mathbb{R}_{\mathcal{C}(M)}$ can be rephrased as follows: a set $S \subseteq \mathbb{R}^{n}$ is $\mathcal{C}(M)$-semianalytic if for each point $p \in \mathbb{R}^{n}$ there is a box $B$ as above containing $p$ in its interior such that $S \cap B$ is a finite union of sets of the form $\left\{x \in B: f(x)=0, g_{1}(x)>0, \ldots, g_{k}(x)>0\right\}$ with $f, g_{1}, \ldots, g_{k} \in \mathcal{C}_{B}(M)$. A set $X \subseteq \mathbb{R}^{m}$ is $\mathcal{C}(M)$-subanalytic if each point in $\mathbb{R}^{m}$ has a neighborhood $U$ such that $X \cap U$ is the image of a bounded $\mathcal{C}(M)$-semianalytic set in $\mathbb{R}^{n}$ for some $n \geq m$ under the projection map $\left(x_{1}, \ldots, x_{n}\right) \mapsto\left(x_{1}, \ldots, x_{m}\right): \mathbb{R}^{n} \rightarrow \mathbb{R}^{m}$. Then Theorem 1 says that the complement of a $\mathcal{C}(M)$-subanalytic set is $\mathcal{C}(M)$-subanalytic. Furthermore, the o-minimality and polynomial boundedness have numerous consequences, such as Łojasiewicz inequalities; see [15] and see [7] for a survey of such consequences.

Our proof of Theorem 1 actually shows (see Theorem [5.2) that the elimination down to only existential quantifiers is "explicit" in the sense of [10] (where a corresponding theorem is proved for real analytic functions).

Furthermore, quasianalytic Denjoy-Carleman classes arising from strongly logconvex sequences are ubiquitous in the following sense (inspired by a theorem of Mandelbrojt [14):

Theorem 2. (1) Given any $C^{\infty}$ function $f: U \longrightarrow \mathbb{R}$, where $U$ is an open neighborhood of $[-1,1]^{n}$ and $n \in \mathbb{N}$, there exist strongly log-convex sequences $M$ and $N$, each satisfying (QA], and functions $f_{1} \in \mathcal{C}_{[-1,1]^{n}}^{0}(M)$ and $f_{2} \in$ $\mathcal{C}_{[-1,1]^{n}}^{0}(N)$ such that $f(x)=f_{1}(x)+f_{2}(x)$ for all $x \in[-1,1]^{n}$.

(2) There exists a strongly log-convex sequence $M$ satisfying (QA) and a function $f \in \mathcal{C}_{[-1,1]}^{0}(M)$ such that $f$ is nowhere analytic.

Combining Theorem 1 with Theorem 2, we answer the two questions from the theory of o-minimal structures posed at the beginning of this introduction:

Corollary. (1) There are strongly log-convex sequences $M$ and $N$, each satisfying (QA), such that $\mathbb{R}_{\mathcal{C}(M)}$ and $\mathbb{R}_{\mathcal{C}(N)}$ are not both reducts of any one o-minimal expansion of the real field. Hence, there is no largest o-minimal expansion of the real field.

(2) There is a strongly log-convex sequence $M$ satisfying $\mathrm{QA}$ such that $\mathbb{R}_{\mathcal{C}(M)}$ does not admit analytic cell decomposition.

Indeed, using Theorem 2(1), we can construct o-minimal expansions $\mathcal{R}_{1}$ and $\mathcal{R}_{2}$ of the real field whose amalgamation defines the set $\mathbb{Z}$ of all integers. 
The idea for the proof of Theorem 1 is as follows. We try to follow the constructions of the o-minimal structures in 8, , 9]. The main ingredients there are a Weierstrass Preparation Theorem due to Tougeron and an adaptation of Gabrielov's fiber cutting argument to the non-Noetherian case. The main problem about transferring these ideas to the situation in this paper is that quasianalytic Denjoy-Carleman classes are not known to satisfy any kind of Weierstrass Preparation Theorem suitable to our purposes (see [5]).

However, Bierstone and Milman's theorem on resolution of singularities [4 applies to our situation and can indeed be used in place of Weierstrass Preparation. In fact, [4] is more general than what is needed here; we only make use of normalization for hypersurfaces. Thus, instead of directly appealing to [4], we use some of the ideas in [3, Section 4] to produce a normalization algorithm for our situation (see Theorem 2.5).

The contents of this paper are organized as follows. To simplify notations (and for easy reference), most of the paper is written under axiomatic assumptions, which are motivated by the closure properties of the Denjoy-Carleman classes discussed above. We briefly present some key points of the Newton algorithm in Section 1, in such a way that our normalization algorithm, developed in Section 2, can readily be seen as a generalization of the two-variable case. In Sections 3 and 4 we combine this algorithm with the fiber cutting arguments of [8]. Finally, we prove Theorem 1 in Section 5 In the appendix, we indicate how the closure properties for the system of all $\mathcal{C}_{B}(M)$ are obtained, and we prove Theorem 2 .

\section{NeWTON ALGORITHM IN TWO VARIABLES}

We recall here some of the ideas of the Newton algorithm for arbitrary real power series of two variables $X$ and $Y$. These ideas will be adapted in Section 2 to the setting described in the introduction.

Let $f(X, Y) \in \mathbb{R} \llbracket X, Y \rrbracket$ be nonzero; we want to use (formal) blowings-up with center the origin to transform $f$ into a normal series, that is, a series of the form $X^{\alpha} Y^{\beta} g(X, Y)$, where $\alpha, \beta \in \mathbb{N}$ and $g \in \mathbb{R} \llbracket X, Y \rrbracket$ is a unit. To do so, we use blow-up substitutions representing the (formal) charts of such a blowing-up: for $\lambda \in \mathbb{R}$ we let $\mathrm{b}_{\lambda}: \mathbb{R} \llbracket X, Y \rrbracket \longrightarrow \mathbb{R} \llbracket X, Y \rrbracket$ be the $\mathbb{R}$-algebra homomorphism defined by $\mathrm{b}_{\lambda}(X):=X$ and $\mathrm{b}_{\lambda}(Y):=X(\lambda+Y)$, and we let $\mathrm{b}_{\infty}: \mathbb{R} \llbracket X, Y \rrbracket \longrightarrow \mathbb{R} \llbracket X, Y \rrbracket$ be the $\mathbb{R}$-algebra homomorphism defined by $\mathrm{b}_{\infty}(X):=X Y$ and $\mathrm{b}_{\infty}(Y):=Y$.

By factoring out a power of $X$, we may assume that $f$ is regular of order $d$ in $Y$, that is, $\operatorname{ord}_{Y} f(0, Y)=d$. Of course, a single application of a blow-up substitution does not in general transform $f$ into a normal series. Instead, we hope that such an application lowers $\operatorname{ord}_{Y} f(0, Y)$; however, in order for such a substitution to represent a meaningful geometric operation (as needed in Section [3), we will need that $\operatorname{ord}_{Y} \mathrm{~b}_{\lambda} f(0, Y)<\operatorname{ord}_{Y} f(0, Y)$ for all $\lambda \in \mathbb{R} \cup\{\infty\}$. This is not always the case, and other kinds of substitutions are needed to handle certain $f$.

For example, assume that the term of $f$ of degree $d-1$ in $Y$ is not zero. Then by the implicit function theorem, there is $\alpha(X) \in \mathbb{R} \llbracket X \rrbracket$ such that $\partial f / \partial Y(X, \alpha(X))=$ 0 . Substituting $Y-\alpha(X)$ for $Y$ (a translation substitution) changes $f$ into a series $g$ such that $\operatorname{ord}_{Y} g(0, Y)=d$ and the term of $g$ of degree $d-1$ in $Y$ is zero. Thus, 
we may assume that

$$
\begin{aligned}
f(X, Y) & =Y^{d} U(X, Y)+\sum_{k \in K} a_{k}(X) Y^{k} \\
& =Y^{d} U(X, Y)+\sum_{k \in K} X^{r_{k}} U_{k}(X) Y^{k},
\end{aligned}
$$

where $K \subseteq\{0, \ldots, d-2\}$ and $U(X, Y) \in \mathbb{R} \llbracket X, Y \rrbracket$ and all $U_{k}(X) \in \mathbb{R} \llbracket X \rrbracket$ are units. Since $f(X, Y)$ is normal if $K=\emptyset$, we assume also that $K \neq \emptyset$. As Section 2 will show, the way we removed the $Y^{d-1}$-term preserves $\operatorname{ord}_{Y} f(0, Y)=d$, so $r_{k} \neq 0$ for all $k \in K$. Further replacing $X$ by $X^{d !}$ (a power substitution), we may also assume that $r_{k}$ is divisible by $d-k$ for each $k \in K$. We now let $l=l(f) \in K$ be maximal such that

$$
\frac{r_{l}}{d-l} \leq \frac{r_{k}}{d-k} \quad \text { for all } k \in K .
$$

In this situation, we claim that the pair $\left(d, r_{l}\right)$ is lowered lexicographically by the application of $\mathrm{b}_{\lambda}$ for any $\lambda \in \mathbb{R} \cup\{\infty\}$ (after possibly factoring out some power of $X)$.

First, we consider the case $\lambda=\infty$. Then

$$
\mathrm{b}_{\infty} f(X, Y)=Y^{d} U(X Y, Y)+\sum_{k \in K} X^{r_{k}} U_{k}(X Y) Y^{k+r_{k}},
$$

and since $k+r_{k} \geq d$ for all $k \in K$, it follows that $\mathrm{b}_{\infty} f$ is normal.

Second, let $\lambda \in \mathbb{R}$ be nonzero. Then

$$
\begin{aligned}
\mathrm{b}_{\lambda} f(X, Y) & =X^{d}\left((\lambda+Y)^{d} U(X, X(\lambda+Y))+\sum_{k \in K} X^{r_{k}-(d-k)} U_{k}(X)(\lambda+Y)^{k}\right) \\
& =X^{d} g(X, Y), \text { say. }
\end{aligned}
$$

Since $d-1 \notin K, g(0, Y)$ has a nonzero coefficient for $Y^{d-1}$, so $\operatorname{ord}_{Y} g(0, Y) \leq d-1$.

Finally, for $\lambda=0$ we have

$$
\mathrm{b}_{0} f(X, Y)=X^{d}\left(Y^{d} U(X, X Y)+\sum_{k \in K} X^{r_{k}-(d-k)} U_{k}(X) Y^{k}\right) .
$$

Now note that $r_{k}-(d-k)$ is divisible by $d-k$ for each $k \in K$. Hence $\mathrm{b}_{0} f=X^{d} g$ with either $\operatorname{ord}_{Y} g(0, Y)<\operatorname{ord}_{Y} f(0, Y)$, or $\operatorname{ord}_{Y} g(0, Y)=\operatorname{ord}_{Y} f(0, Y)$ and $r_{l(g)}<r_{l(f)}$.

In the situation where $X=\left(X_{1}, \ldots, X_{n}\right)$ is a tuple of variables rather than a single variable, the property of having no term of degree $d-1$ in $Y$ is not sufficient to write $f$ as in (1.1). Instead, if

$$
f(X, Y)=Y^{d} U(X, Y)+\sum_{k \in K} a_{k}(X) Y^{k}
$$

with $K \subseteq\{0, \ldots, d-2\}, U \in \mathbb{R} \llbracket X, Y \rrbracket$ a unit and $a_{k}(X) \in \mathbb{R} \llbracket X \rrbracket$ nonunits different from 0 , we first need to work inductively on the $a_{k}(X)$ 's to reduce them to the form $a_{k}(X)=X^{r_{k}} U_{k}(X)$, such that $r_{k} \in \mathbb{N}^{n}$ and $U_{k}(X) \in \mathbb{R} \llbracket X \rrbracket$ is a unit for each $k \in K$ and, in addition, the monomials $X^{r_{k}}$ are linearly ordered by divisibility (Step 2 of Section (2). 


\section{A NORMALIZATION ALGORITHM}

Let $X=\left(X_{1}, \ldots, X_{n}\right)$, and write $X^{\prime}:=\left(X_{1}, \ldots, X_{n-1}\right)$ if $n>0$.

Definition 2.1. A series $f \in \mathbb{R} \llbracket X \rrbracket$ is called normal if $f(X)=X^{r} \cdot U(X)$ with $r \in \mathbb{N}^{n}$ and $U \in \mathbb{R} \llbracket X \rrbracket$ a unit. (This terminology has its origin in "normal crossings", as used for instance in [4].)

A set $\left\{f_{1}, \ldots, f_{l}\right\} \subseteq \mathbb{R} \llbracket X \rrbracket$ of series is normal if $f_{k}(X)=X^{r_{k}} U_{k}(X)$, with $r_{k} \in \mathbb{N}^{n}$ and $U_{k}$ a unit for each $k$, and the set of monomials $\left\{X^{r_{1}}, \ldots, X^{r_{l}}\right\}$ is linearly ordered by divisibility. (The divisibility relation for monomials $X^{\alpha}$ with $\alpha \in \mathbb{R}^{n}$ is given by the product ordering on their exponents $\alpha$.)

The following observations are elementary (see for instance [3, Lemma 4.7].)

Lemma 2.2. (1) Let $f_{1}, \ldots, f_{l} \in \mathbb{R} \llbracket X \rrbracket$. Then $f_{1} \cdots f_{l}$ is normal if and only if each $f_{k}$ is normal.

(2) Let $f_{1}, \ldots, f_{l} \in \mathbb{R} \llbracket X \rrbracket$, and assume that all $f_{k}$, for $k=1, \ldots, l$, and all $f_{k}-f_{k^{\prime}}$, for $0 \leq k<k^{\prime} \leq l$, are normal. Then $\left\{f_{1}, \ldots, f_{l}\right\}$ is normal.

For $1 \leq i \leq n$ and an integer $q>0$ we let $p_{i, q}^{+}, p_{i, q}^{-}: \mathbb{R} \llbracket X \rrbracket \longrightarrow \mathbb{R} \llbracket X \rrbracket$ be the $\mathbb{R}$-algebra homomorphisms defined by

$$
p_{i, q}^{+}\left(X_{j}\right):= \begin{cases}X_{i}^{q} & \text { if } j=i \\ X_{j} & \text { otherwise }\end{cases}
$$

and

$$
p_{i, q}^{-}\left(X_{j}\right):= \begin{cases}-X_{i}^{q} & \text { if } j=i, \\ X_{j} & \text { otherwise. }\end{cases}
$$

Also, for $1 \leq i \leq n$ and $\alpha \in \mathbb{R} \llbracket X_{1}, \ldots, X_{i-1} \rrbracket$ such that $\alpha(0)=0$, we let $t_{\alpha}$ : $\mathbb{R} \llbracket X \rrbracket \longrightarrow \mathbb{R} \llbracket X \rrbracket$ be the $\mathbb{R}$-algebra homomorphism given by

$$
t_{\alpha}\left(X_{j}\right):= \begin{cases}X_{i}+\alpha\left(X_{1}, \ldots, X_{i-1}\right) & \text { if } j=i, \\ X_{j} & \text { otherwise. }\end{cases}
$$

Next, for $i>1$ and $c=\left(c_{1}, \ldots, c_{i-1}\right) \in \mathbb{R}^{i-1}$, we let $l_{i, c}: \mathbb{R} \llbracket X \rrbracket \longrightarrow \mathbb{R} \llbracket X \rrbracket$ be the $\mathbb{R}$-algebra homomorphism given by

$$
l_{i, c}\left(X_{j}\right):= \begin{cases}X_{j}+c_{j} X_{i} & \text { if } 1 \leq j<i, \\ X_{j} & \text { otherwise. }\end{cases}
$$

Finally, for $1 \leq i<j \leq n$ and $\lambda \in \mathbb{R}$, we let $\mathrm{b}_{\lambda}^{i, j}: \mathbb{R} \llbracket X \rrbracket \longrightarrow \mathbb{R} \llbracket X \rrbracket$ be the $\mathbb{R}$-algebra homomorphism given by

$$
\mathrm{b}_{\lambda}^{i, j}\left(X_{k}\right):= \begin{cases}X_{i}\left(\lambda+X_{j}\right) & \text { if } k=j \\ X_{k} & \text { otherwise }\end{cases}
$$

and we let $\mathrm{b}_{\infty}^{i, j}: \mathbb{R} \llbracket X \rrbracket \longrightarrow \mathbb{R} \llbracket X \rrbracket$ be the $\mathbb{R}$-algebra homomorphism defined by

$$
\mathrm{b}_{\infty}^{i, j}\left(X_{k}\right):= \begin{cases}X_{i} X_{j} & \text { if } k=i \\ X_{k} & \text { otherwise. }\end{cases}
$$

For the remainder of this section, we fix an arbitrary family $\mathcal{D}=\left(\mathcal{D}_{n}\right)_{n \in \mathbb{N}}$, where each $\mathcal{D}_{n}$ is an $\mathbb{R}$-subalgebra of $\mathbb{R} \llbracket X \rrbracket$ containing $\mathbb{R}[X]$ as a subalgebra. 
Definition 2.3. A $\mathcal{D}$-admissible substitution is any one of the following collections of $\mathbb{R}$-algebra homomorphisms $\mathbb{R} \llbracket X \rrbracket \longrightarrow \mathbb{R} \llbracket X \rrbracket$ :

(1) $\tau=\left\{l_{i, c}\right\}$ for some $1<i \leq n$ and $c \in \mathbb{R}^{i-1}$ (a linear substitution);

(2) $\tau=\left\{t_{\alpha}\right\}$ for some $1<i \leq n$ and $\alpha \in \mathcal{D}_{i-1}$ with $\alpha(0)=0$ (a translation substitution);

(3) $\tau=\left\{p_{i, q}^{+}, p_{i, q}^{-}\right\}$for some $1 \leq i \leq n$ and integer $q>0$ (a power substitution);

(4) $\tau=\mathrm{b}^{i, j}=\left\{\mathrm{b}_{\lambda}^{i, j}: \lambda \in \mathbb{R} \cup\{\infty\}\right\}$ for some $1 \leq i<j \leq n$ (a blow-up substitution).

If $\mathcal{D}$ is clear from context, we shall simply refer to $\mathcal{D}$-admissible substitutions as admissible.

Remark. The choice of the collections of homomorphisms above is due to the fact that when the corresponding substitutions are interpreted as geometric operations in Section [3, the members of such a collection will represent charts of the same geometric operation.

We assume from now on that

(Q1) $\mathcal{D}_{n} \subseteq \mathcal{D}_{n+1}$ for all $n$;

(Q2) if $f \in \mathcal{D}_{m}$ and $g_{1}, \ldots, g_{m} \in \mathcal{D}_{n}$ such that $g_{1}(0)=\cdots=g_{m}(0)=0$, then $f\left(g_{1}, \ldots, g_{m}\right) \in \mathcal{D}_{n}$

(Q3) each $\mathcal{D}_{n}$ is closed under taking partial derivatives;

(Q4) if $f \in \mathcal{D}_{n}$ and $g \in \mathbb{R} \llbracket X \rrbracket$ are such that $f(X)=X_{i} \cdot g(X)$ for some $i \leq n$, then $g \in \mathcal{D}_{n}$;

(Q5) if $n>0$ and $f \in \mathcal{D}_{n}$ are such that $f(0)=0$ and $\left(\partial f / \partial X_{n}\right)(0) \neq 0$, there is an $\alpha \in \mathcal{D}_{n-1}$ with $\alpha(0)=0$ such that $f\left(X^{\prime}, \alpha\left(X^{\prime}\right)\right)=0$.

Remarks 2.4. (1) Let $\tau$ be an admissible substitution. Then $f \in \mathbb{R} \llbracket X \rrbracket$ is a unit if and only if every member of $\tau f$ is a unit. Moreover, if $f \in \mathbb{R} \llbracket X \rrbracket$ is normal and $\tau$ is a power substitution or a blow-up substitution, then every member of $\tau f$ is normal.

(2) It follows from assumptions (Q2) and (Q4) that if $f \in \mathcal{D}_{n}$ and

$$
f(X)=\left(X_{i}-a\left(X_{1}, \ldots, X_{i-1}\right)\right) g(X)
$$

with $g \in \mathbb{R} \llbracket X \rrbracket$ and $1 \leq i \leq n, a \in \mathcal{D}_{i-1}$ and $a(0)=0$, then $g \in \mathcal{D}_{n}$.

(3) The following observation will be used in Section 3 In the situation of (Q5), there is for each nonzero $\mu \in \mathbb{N}^{n-1}$ a positive integer $k$ such that $\alpha^{(\mu)} \in u^{-k} R$, where $R:=\mathbb{Z}\left[f^{(\nu)}\left(X^{\prime}, \alpha\left(X^{\prime}\right)\right): \nu \in \mathbb{N}^{n}\right] \subseteq \mathcal{D}_{n-1}$ and $u:=\left(\partial f / \partial X_{n}\right)\left(X^{\prime}, \alpha\left(X^{\prime}\right)\right)$, a unit of $\mathcal{D}_{n-1}$.

(4) It follows from (Q5) that if $f \in \mathcal{D}_{n}$ and $f(0) \neq 0$, then $f$ is a unit in $\mathcal{D}_{n}$.

We will define a map $\mathrm{h}_{n}: \mathcal{D}_{n} \backslash\{0\} \longrightarrow(\mathbb{N} \cup\{\infty\})^{\nu_{n}}$ for $n \geq 1$, where $\nu_{n}$ only depends on $n$, such that $\mathrm{h}_{n}(f)=(0, \ldots, 0)$ implies that $f$ is normal. (To simplify notation, we usually write 0 in place of $(0, \ldots, 0)$.) Equipping $(\mathbb{N} \cup\{\infty\})^{\nu_{n}}$ with the lexicographic ordering, we could say that $\mathrm{h}_{n}(f)$ measures "how far from normal" $f$ is. More precisely, we prove the following.

Theorem 2.5. Let $n \geq 1$ and $f \in \mathcal{D}_{n}$ be nonzero.

(1) If $\mathrm{h}_{n}(f)=0$, then $f$ is normal. 
(2) If $\mathrm{h}_{n}(f)>0$, then there is an admissible substitution $\tau$ such that $\mathrm{h}_{n}(g)<$ $\mathrm{h}_{n}(f)$ for all $g \in \tau f$.

We prove the theorem by induction on $n \geq 1$; since every nonzero $f \in \mathcal{D}_{1}$ is normal, we can take $\nu_{1}:=1$ and $\mathrm{h}_{1}(f):=0$ for all nonzero $f \in \mathcal{D}_{1}$. So we assume for the remainder of this section that $n>1$ and that $\mathrm{h}_{1}, \ldots, \mathrm{h}_{n-1}$ are defined and have the desired properties.

For nonzero $f \in \mathcal{D}_{n}$ we let

$$
\mathcal{F}_{f}:=\left\{(h, g): h \in \mathcal{D}_{n-1}, g \in \mathcal{D}_{n} \text { and } f(X)=h\left(X^{\prime}\right) \cdot g(X)\right\},
$$

and we define

$$
\operatorname{ord}_{n}(f):=\min \left\{\operatorname{ord}_{X_{n}} g\left(0, X_{n}\right):(h, g) \in \mathcal{F}_{f} \text { for some } h \in \mathcal{D}_{n-1}\right\} ;
$$

so $\operatorname{ord}_{n}(f) \in \mathbb{N} \cup\{\infty\}$. By Remark 2.4(4), any nonzero $f \in \mathcal{D}_{n}$ with $\operatorname{ord}_{n}(f)=0$ is of the form $h \cdot U$ with $h \in \mathcal{D}_{n-1}$ and $U \in \mathcal{D}_{n}$ a unit. Thus, the main idea is to lower $\operatorname{ord}_{n}(f)$ until it reaches 0 . To do so, we proceed in several steps (Steps 1 through 4 below); for $m=1,2,3,4$, we define in Step $m$ a property $P_{m}$ of $f$ and a tuple $\mathrm{i}_{m}(f) \in(\mathbb{N} \cup\{\infty\})^{\mu_{m}}$ (where $\mu_{m}$ only depends on $m$ and $n$ and each $(\mathbb{N} \cup\{\infty\})^{\mu_{m}}$ is considered with the corresponding lexicographic ordering) to measure "how far $f$ is from having property $P_{m}$ ". In turn, properties $P_{1}, \ldots, P_{4}$ express (with increasing accuracy) how far $f$ is from the situation where we can lower $\operatorname{ord}_{n}(f)$ with a blow-up substitution.

First, our definition of $\mathrm{i}_{m}(f)$ will imply that for any nonzero $f \in \mathcal{D}_{n}$,

$$
\operatorname{ord}_{n}(f)=0 \quad \Longrightarrow \quad \mathrm{i}_{1}(f)=\mathrm{i}_{3}(f)=\mathrm{i}_{4}(f)=0 \text { and } \mathrm{i}_{2}(f)=\mathrm{h}_{n-1}(h),
$$

where $\mathrm{h}_{n-1}(h)$ is minimal among all $h \in \mathcal{D}_{n-1}$ for which there is a unit $U(X) \in \mathcal{D}_{n}$ such that $(h, U) \in \mathcal{F}_{f}$.

Second, we prove

Proposition 2.6. Let $f \in \mathcal{D}_{n}$ be nonzero such that $0<\operatorname{ord}_{n}(f)<\infty$ and $m \in$ $\{1,2,3\}$.

(1) If $\mathrm{i}_{1}(f)=\cdots=\mathrm{i}_{m}(f)=0$, then $f$ has property $P_{m}$.

(2) If $\mathrm{i}_{1}(f)=\cdots=\mathrm{i}_{m-1}(f)=0$ and $\mathrm{i}_{m}(f)>0$, there is an admissible substitution $\tau$ such that for all $\phi \in \tau f$, we have $\operatorname{ord}_{n}(\phi) \leq \operatorname{ord}_{n}(f)$, $\mathrm{i}_{1}(\phi)=\cdots=\mathrm{i}_{m-1}(\phi)=0$ and $\mathrm{i}_{m}(\phi)<\mathrm{i}_{m}(f)$.

Third, similar to the arguments presented in Section 1, we prove

Proposition 2.7. Let $f \in \mathcal{D}_{n}$ be nonzero such that $f$ is not normal and $0<$ $\operatorname{ord}_{n}(f)<\infty$. If $\mathrm{i}_{1}(f)=\mathrm{i}_{2}(f)=\mathrm{i}_{3}(f)=0$, there is a blow-up substitution $\tau$ such that for every $\phi \in \tau f$,

(1) either $\operatorname{ord}_{n}(\phi)<\operatorname{ord}_{n}(f)$

(2) or $\mathrm{i}_{1}(\phi)=\mathrm{i}_{2}(\phi)=\mathrm{i}_{3}(\phi)=0, \operatorname{ord}_{n}(\phi)=\operatorname{ord}_{n}(f)$ and $\mathrm{i}_{4}(\phi)<\mathrm{i}_{4}(f)$.

Finally, we define for nonzero $f \in \mathcal{D}_{n}$,

$$
\mathrm{h}_{n}(f):=\left(\operatorname{ord}_{n}(f), \mathrm{i}_{1}(f), \ldots, \mathrm{i}_{4}(f)\right) .
$$

Assuming (2.1) and Propositions 2.6 and 2.7 we finish the proof of Theorem 2.5 as follows. Note that if $f \in \mathcal{D}_{n} \backslash\{0\}$ is such that $\operatorname{ord}_{n}(f)=\infty$, there is a $c \in \mathbb{R}^{n-1}$ such that $\operatorname{ord}_{n}\left(l_{n, c} f\right)<\infty$; so we assume that $\operatorname{ord}_{n}(f)<\infty$. By Propositions 2.6 and 2.7. we may then even assume that $\operatorname{ord}_{n}(f)=0$, that is, $f(X)=h\left(X^{\prime}\right) U(X)$ with $h \in \mathcal{D}_{n-1}, U \in \mathcal{D}_{n}$ a unit and $\mathrm{h}_{n-1}(h)$ minimal. Then by (2.1), we have 
$\mathrm{h}_{n}(f)=\left(0, \mathrm{~h}_{n-1}(h), 0,0\right)$; thus, if $\mathrm{h}_{n}(f)=0$, then $\mathrm{h}_{n-1}(h)=0$ and $f$ is normal by the inductive hypothesis, so we assume that $\mathrm{h}_{n}(f) \neq 0$. Again by the inductive hypothesis, there is an admissible substitution $\tau$ (in the variables $X^{\prime}$ ) such that $\mathrm{h}_{n-1}(g)<\mathrm{h}_{n-1}(h)$ for all $g \in \tau h$. On the other hand, since every $\phi \in \tau f$ is of the form $g W$ for some $g \in \tau h$ and $W \in \tau U$, it follows from Remark 2.4(1) and (2.1) that $\mathrm{h}_{n}(\phi)<\mathrm{h}_{n}(f)$ for all $\phi \in \tau f$. This then finishes the proof of Theorem 2.5 .

Therefore, it remains to prove Propositions 2.6 and 2.7 and verify (2.1).

Step 1. A nonzero $f \in \mathcal{D}_{n}$ has property $P_{1}$ if $d:=\operatorname{ord}_{n}(f)<\infty$ and there are $(h, g) \in \mathcal{F}_{f}$ and $K \subseteq\{0, \ldots, d-2\}$ such that

(*) $g(X)=X_{n}^{d} U(X)+\sum_{k \in K} g_{k}\left(X^{\prime}\right) X_{n}^{k}$, with each $g_{k}\left(X^{\prime}\right) \in \mathcal{D}_{n-1} \backslash\{0\}$ a nonunit and $U(X) \in \mathcal{D}_{n}$ a unit.

(Note that there is no term of degree $d-1$ in $X_{n}$.) For nonzero $g \in \mathcal{D}_{n}$ we put $\mathrm{i}_{1}^{0}(g):=0$ if $g$ is of the form $(*)$ for some $d \in \mathbb{N}$ and $\mathrm{i}_{1}^{0}(g):=1$ otherwise. Then for nonzero $f \in \mathcal{D}_{n}$ we put

$$
\begin{aligned}
\mathrm{i}_{1}(f):=\min \left\{\mathrm{i}_{1}^{0}(g):(h, g) \in \mathcal{F}_{f} \text { for some } h\right. & \in \mathcal{D}_{n-1} \\
& \text { and } \left.\operatorname{ord}_{X_{n}} g\left(0, X_{n}\right)=\operatorname{ord}_{n}(f)\right\} .
\end{aligned}
$$

Note that $\mathrm{i}_{1}(f)=0$ if $\operatorname{ord}_{n}(f)=0$, as required for 2.1).

Proof of Proposition 2.6 for $m=1$. Let $f \in \mathcal{D}_{n}$ be such that $\operatorname{ord}_{n}(f)=d<\infty$, and let $(h, g) \in \mathcal{F}_{f}$ be such that $\operatorname{ord}_{X_{n}} g\left(0, X_{n}\right)=d$. If $d=0$, there is nothing to prove, so we assume that $d>0$. Considering $g(X)$ as a series in $\mathcal{D}_{n+1}$, we see by a formal Taylor expansion in powers of $X_{n}-X_{n+1}$ around $X_{n+1}$ that there is a series $U\left(X, X_{n+1}\right) \in \mathcal{D}_{n+1}$ such that

$$
g(X)=\left(X_{n}-X_{n+1}\right)^{d} U\left(X, X_{n+1}\right)+\sum_{k=0}^{d-1} \frac{\partial^{k} g}{\partial X_{n}^{k}}\left(X^{\prime}, X_{n+1}\right) \frac{\left(X_{n}-X_{n+1}\right)^{k}}{k !} .
$$

Since $\operatorname{ord}_{X_{n}} g\left(0, X_{n}\right)=d$, it follows that $\left(\partial^{k} g / \partial X_{n}^{k}\right)(0)=0$ for all $k<d$, while $\left(\partial^{d} g / \partial X_{n}^{d}\right)(0) \neq 0$; in particular, $U$ is a unit. By assumption (Q5) there exists $a=a(g) \in \mathcal{D}_{n-1}$ such that $a(0)=0$ and $\left(\partial^{d-1} g / \partial X_{n}^{d-1}\right)\left(X^{\prime}, a\left(X^{\prime}\right)\right)=0$. Evaluating $g(X)$ at $X_{n+1}=a\left(X^{\prime}\right)$ (a procedure corresponding to the Tschirnhausen transformation (see [1, 2])) gives

$$
g(X)=\left(X_{n}-a\left(X^{\prime}\right)\right)^{d} U\left(X, a\left(X^{\prime}\right)\right)+\sum_{k=0}^{d-2} \frac{\partial^{k} g}{\partial X_{n}^{k}}\left(X^{\prime}, a\left(X^{\prime}\right)\right) \frac{\left(X_{n}-a\left(X^{\prime}\right)\right)^{k}}{k !} .
$$

By Remark $2.4(3)$, we have $U\left(X, a\left(X^{\prime}\right)\right) \in \mathcal{D}_{n}$, while $\operatorname{ord}_{n}\left(t_{a} f\right)=\operatorname{ord}_{n}(f)$ and $\mathrm{i}_{1}\left(t_{a} f\right)=0$.

Step 2. A nonzero $f \in \mathcal{D}_{n}$ has property $P_{2}$ if $d:=\operatorname{ord}_{n}(f)<\infty$ and there are $(h, g) \in \mathcal{F}_{f}$ and $K \subseteq\{0, \ldots, d-2\}$ such that

$(* *) g$ satisfies $(*)$, and $h$ and the set $\left\{g_{k}^{d ! /(d-k)}: k \in K\right\}$ are normal.

Let $f \in \mathcal{D}_{n}$ be nonzero. If $d:=\operatorname{ord}_{n}(f)=\infty$, we put $\mathcal{F}_{f}\left(P_{1}\right):=\emptyset$, and if $d<\infty$, we let $\mathcal{F}_{f}\left(P_{1}\right)$ be the set of all $(h, g) \in \mathcal{F}_{f}$ for which $(*)$ holds. Also, if $f$ has property $P_{1}$ and $(h, g) \in \mathcal{F}_{f}\left(P_{1}\right)$, we let $\widetilde{g} \in \mathcal{D}_{n-1}$ be the product of $h$ with all 
$g_{k}^{d ! /(d-k)}, k \in K$, and all nonzero $g_{k}^{d ! /(d-k)}-g_{l}^{d ! /(d-l)}$ with $k, l \in K$ such that $k<l$ (where $K$ and the $g_{k}$ are associated to $g$ as in $(*)$ ). In this situation we define

$$
\mathrm{i}_{2}(f):=\min \left\{\mathrm{h}_{n-1}(\widetilde{g}):(h, g) \in \mathcal{F}_{f}\left(P_{1}\right)\right\} .
$$

(If $f \in \mathcal{D}_{n} \backslash\{0\}$ does not have property $P_{1}$, we set $\mathrm{i}_{2}(f):=\infty$.) Note that if $\operatorname{ord}_{n}(f)=0$, there is an $h\left(X^{\prime}\right) \in \mathcal{D}_{n-1}$ such that $(h, U) \in \mathcal{F}_{f}$ for some unit $U(X) \in$ $\mathcal{D}_{n}$ and $\mathrm{h}_{n-1}(h)$ is minimal. Thus $\widetilde{g}=h$ in this situation, so $\mathrm{i}_{2}(f)=\mathrm{h}_{n-1}(h)$, as required for 2.1.

Proof of Proposition [2.6 for $m=2$. Part (1) is immediate, so let $f \in \mathcal{D}_{n}$ be nonzero such that $\mathrm{i}_{1}(f)=0$ and $\mathrm{i}_{2}(f)>0$. Let $(h, g) \in \mathcal{F}_{f}\left(P_{1}\right)$ be such that $\mathrm{i}_{2}(f)=\mathrm{h}_{n-1}(\widetilde{g})$. By the inductive hypothesis, there is an admissible substitution $\tau$ (in the variables $\left.X^{\prime}\right)$ such that $\mathrm{h}_{n-1}(\theta)<\mathrm{h}_{n-1}(\widetilde{g})$ for all $\theta \in \tau \widetilde{g}$. Arguing as in the proof of Theorem 2.5 above, we see that $\operatorname{ord}_{n}(\phi) \leq \operatorname{ord}_{n}(f), \mathrm{i}_{1}(\phi)=0$ and $\mathrm{i}_{2}(\phi)<\mathrm{i}_{2}(f)$ for all $\phi \in \tau f$.

Remark 2.8. If $f \in \mathcal{D}_{n}$ has property $P_{2}$, there are unique $\alpha=\alpha_{f} \in \mathbb{N}^{n-1}$ and $g=g_{f} \in \mathcal{D}_{n}$ such that $d:=\operatorname{ord}_{n}(f)=\operatorname{ord}_{X_{n}} g\left(0, X_{n}\right)<\infty$ and $\left(\left(X^{\prime}\right)^{\alpha}, g\right)$ belongs to $\mathcal{F}_{f}$ and satisfies $(* *)$. If, in addition, the corresponding $K=K_{f} \subseteq\{0, \ldots, d-2\}$ is empty, then $f$ is normal. On the other hand, if $K \neq \emptyset$, then for each $k \in K$, there are $r_{k}=\left(r_{k, 1}, \ldots, r_{k, n-1}\right) \in \mathbb{N}^{n-1}$ and a unit $U_{k} \in \mathcal{D}_{n-1}$ such that $g_{k}=$ $\left(X^{\prime}\right)^{r_{k}} U_{k}\left(X^{\prime}\right)$. Moreover, for every integer $p>0$ and every $i \in\{1, \ldots, n-1\}$, the set $\left\{\left(X^{\prime}\right)^{r_{k}^{i} /(d-k)}: k \in K\right\}$ of monomials (with possibly rational exponents) is totally ordered by divisibility, where $r_{k}^{i}=\left(r_{k, 1}^{i}, \ldots, r_{k, n-1}^{i}\right), r_{k, i}^{i}:=p r_{k, i}$ and $r_{k, j}^{i}:=r_{k, j}$ for $j \neq i$.

Step 3. Let $f \in \mathcal{D}_{n}$ have property $P_{2}$, and let $d, \alpha, K$ and $g$, etc., be as in Remark 2.8. In this situation, we say that $f$ has property $P_{3}$ if $r_{k, i}$ is divisible by $d-k$ for all $k$ and $i$. Thus we put

$$
B(f):=\left\{i<n: r_{k, i} \text { is not divisible by } d-k \text { for some } k \in K\right\}
$$

and define $\mathrm{i}_{3}(f):=|B(f)|$. (If $f \in \mathcal{D}_{n} \backslash\{0\}$ does not have property $P_{2}$, we set $\mathrm{i}_{3}(f):=\infty$.) Note that if $K=\emptyset$ (and hence in particular if $\operatorname{ord}_{n}(f)=0$ ), then $B(f)=\emptyset$, so $\mathrm{i}_{3}(f)=0$ as well, as required for (2.1).

Proof of Proposition [2.6 for $m=3$. Let $f \in \mathcal{D}_{n}$ be such that $\mathrm{i}_{1}(f)=\mathrm{i}_{2}(f)=0$ and $\mathrm{i}_{3}(f)>0$, and let $B(f)$ be as above. Choose any $i \in B(f)$ and put $\tau:=\left\{p_{i, d !}^{+}, p_{i, d !}^{-}\right\}$. Then for all $\phi \in \tau f$ we have $\operatorname{ord}_{n}(\phi)=\operatorname{ord}_{n}(f), \mathrm{i}_{1}(f)=\mathrm{i}_{2}(\phi)=0$ (by Remarks 2.4(1) and 2.8 with $p=d !)$ and $\mathrm{i}_{3}(\phi)<\mathrm{i}_{3}(f)$.

Remark 2.9. Let $q \geq 0$ be an integer. In the situation of the previous proof, note that $\tau\left(X_{i}^{q} f\right)=X_{i}^{q \cdot d !} \tau f$. Hence for all $\phi \in \tau\left(X_{i}^{q} f\right)$ we have $\operatorname{ord}_{n}(\phi)=\operatorname{ord}_{n}(f)$, $\mathrm{i}_{1}(\phi)=\mathrm{i}_{2}(\phi)=0$ and $\mathrm{i}_{3}(\phi)<\mathrm{i}_{3}(f)$.

Step 4. Let $f \in \mathcal{D}_{n}$ have property $P_{3}$; we continue using the corresponding notation of Step 3. If $K \neq \emptyset$ (i.e., $f$ is not already normal), we now want to use a blow-up substitution to lower $\operatorname{ord}_{n}(f)$. However, this only works if for some $k \in K$, we have $r_{k, i}=d-k$ for some $i<n$ and $r_{k, j}=0$ for all $j \neq i$. Thus, we say that $f$ has property $P_{4}$ if either $K=\emptyset$ or there is such a $k \in K$, and we define $\mathrm{i}_{4}(f)$ as follows: if $K \neq \emptyset$, we let $l=l(f) \in K$ be maximal such that $r_{l} /(d-l) \leq r_{k} /(d-k)$ 
for all $k \in K$, and let $j=j(f)<n$ be maximal such that $r_{l, j} \neq 0$. Then we define

$$
\mathrm{i}_{4}(f):= \begin{cases}\left(j, r_{l, j}\right) & \text { if } K \neq \emptyset, \\ (0,0) & \text { otherwise. }\end{cases}
$$

(We put $\mathrm{i}_{4}(\phi):=(\infty, \infty)$ if $f \in \mathcal{D}_{n} \backslash\{0\}$ does not have property $P_{3}$.) Note that $\mathrm{i}_{4}(f) \neq 0$ if $K \neq \emptyset$, while $\mathrm{i}_{4}(f)=0$ if $\operatorname{ord}_{n}(f)=0$.

The proof of Proposition 2.7 is given by the following three lemmas (generalizing the observations in Section 1). We fix a nonzero $f \in \mathcal{D}_{n}$ such that $f$ has property $P_{3}$ and $K \neq \emptyset$; in particular, $\operatorname{ord}_{n}(f)>0$ and $\mathrm{i}_{4}(f)>(0,0)$. Below we continue using the notation associated to $f$ in Remark 2.8 and Step 3. We let $\tau:=\mathrm{b}^{j, n}$ with $j=j(f)$; the three lemmas below actually show that Proposition 2.7 follows for this $\tau$.

Lemma 2.10. Let $\lambda \in \mathbb{R} \backslash\{0\}$. Then $\operatorname{ord}_{n}\left(b_{\lambda}^{j, n} f\right)<\operatorname{ord}_{n}(f)$; in fact, for every integer $q \geq 0$ we have $\operatorname{ord}_{n}\left(\mathrm{~b}_{\lambda}^{j, n}\left(X_{j}^{q} f\right)\right)<\operatorname{ord}_{n}(f)$.

Proof. Since

$$
\mathrm{b}_{\lambda}^{j, n} g(X)=X_{j}^{d}\left(\lambda+X_{n}\right)^{d} \mathrm{~b}_{\lambda}^{j, n} U(X)+\sum_{k \in K}\left(X^{\prime}\right)^{r_{k}} U_{k}\left(X^{\prime}\right) X_{j}^{k}\left(\lambda+X_{n}\right)^{k},
$$

and since $r_{k, j} /(d-k) \geq r_{l, j} /(d-l)>0$ for each $k \in K$, we can factor out $X_{j}^{d}$. Thus for every integer $q \geq 0$, we have $\mathrm{b}_{\lambda}^{j, n}\left(X_{j}^{q} f(X)\right)=\left(X^{\prime}\right)^{\alpha} X_{j}^{q+d} h(X)$ with

$$
h(X)=\left(\lambda+X_{n}\right)^{d} \mathrm{~b}_{\lambda}^{j, n} U(X)+\sum_{k \in K}\left(X^{\prime}\right)^{r_{k}^{\prime}} U_{k}\left(X^{\prime}\right)\left(\lambda+X_{n}\right)^{k},
$$

where $r_{k, j}^{\prime}:=r_{k, j}-(d-k)$ and $r_{k, i}^{\prime}:=r_{k, i}$ if $i \neq j$, for all $k \in K$. Since the only term of $h$ that contributes to the coefficient of $X_{n}^{d-1}$ is $\mathrm{b}_{\lambda}^{j, n} U(X)\left(\lambda+X_{n}\right)^{d}$, and since $\mathrm{b}_{\lambda}^{j, n} U\left(0, \ldots, 0, X_{n}\right)=U(0)$, it follows that $\operatorname{ord}_{n}\left(\mathrm{~b}_{\lambda}^{j, n}\left(X_{j}^{q} f\right)\right) \leq d-1$.

Remark. A careful examination of the previous proof actually shows the following (which we do not need here): for all but finitely many $\lambda \in \mathbb{R}$ the series $b_{\lambda}^{j, n} f$ is normal.

Lemma 2.11. The series $\mathrm{b}_{\infty}^{j, n} f$ is normal. In fact, for every integer $q \geq 0$ the series $\mathrm{b}_{\infty}^{j, n}\left(X_{n}^{q} f\right)$ is normal.

Proof. We have

$$
\mathrm{b}_{\infty}^{j, n} g(X)=\mathrm{b}_{\infty}^{j, n} U(X) X_{n}^{d}+\sum_{k \in K}\left(X^{\prime}\right)^{r_{k}} \mathrm{~b}_{\infty}^{j, n} U_{k}\left(X^{\prime}\right) X_{n}^{k+r_{k, j}} .
$$

Since $r_{k, j} /(d-k) \geq r_{l, j} /(d-l)>0$ for each $k$, we can factor out $X_{n}^{d}$. Thus for every integer $q \geq 0$, we have $\mathrm{b}_{\infty}^{j, n}\left(X_{n}^{q} f(X)\right)=\left(X^{\prime}\right)^{\alpha} X_{n}^{q+d} h(X)$ with

$$
h(X)=\mathrm{b}_{\infty}^{j, n} U(X)+\sum_{k \in K}\left(X^{\prime}\right)^{r_{k}} \mathrm{~b}_{\infty}^{j, n} U_{k}\left(X^{\prime}\right) X_{n}^{k+r_{k, j}-d} .
$$

Since $r_{k}>(0, \ldots, 0)$ for each $k \in K$, it follows that $h$ is a unit.

Lemma 2.12. Let $q \geq 0$ be an integer and write $\phi_{q}:=\mathrm{b}_{0}^{j, n}\left(X_{j}^{q} f\right)$. Then either $\operatorname{ord}_{n}\left(\phi_{q}\right)<\operatorname{ord}_{n}(f)$, or $\operatorname{ord}_{n}\left(\phi_{q}\right)=\operatorname{ord}_{n}(f)$ and $\mathrm{i}_{4}\left(\phi_{q}\right)<\mathrm{i}_{4}(f)$. 
Proof. Note that

$$
\mathrm{b}_{0}^{j, n} g(X)=\mathrm{b}_{0}^{j, n} U(X) X_{j}^{d} X_{n}^{d}+\sum_{k \in K}\left(X^{\prime}\right)^{r_{k}} U_{k}\left(X^{\prime}\right) X_{j}^{k} X_{n}^{k}
$$

As in the proof of Lemma 2.10, for any positive integer $q \geq 0$ we obtain that $\mathrm{b}_{0}^{j, n}\left(X_{j}^{q} f(X)\right)=\left(X^{\prime}\right)^{\alpha} X_{j}^{q+d} h(X)$, where

$$
h(X)=\mathrm{b}_{0}^{j, n} U(X) X_{n}^{d}+\sum_{k \in K}\left(X^{\prime}\right)^{r_{k}^{\prime}} U_{k}\left(X^{\prime}\right) X_{n}^{k}
$$

and $r_{k, j}^{\prime}:=r_{k, j}-(d-k)$ and $r_{k, i}^{\prime}:=r_{k, i}$ if $i \neq j$, for all $k \in K$; in particular, $\operatorname{ord}_{n}\left(\mathrm{~b}_{0}^{j, n}\left(X_{j}^{q} f(X)\right)\right)=\operatorname{ord}_{n}\left(\mathrm{~b}_{0}^{j, n} f(X)\right) \leq \operatorname{ord}_{n}(f)$. Since $r_{k, j}$ is divisible by $d-k$, we see that $\left\{\left(X^{\prime}\right)^{r_{k}^{\prime} /(d-k)}: k \in K\right\}$ is again linearly ordered by divisibility and that $r_{l}^{\prime} /(d-l) \leq r_{k}^{\prime} /(d-k)$ for all $k \in K$.

Assume now that $\operatorname{ord}_{n}(h)=\operatorname{ord}_{n}(f)$; then $r_{l}^{\prime}>(0, \ldots, 0)$. If $r_{l, j}^{\prime} \neq 0$, then $j(h)=j(f)$, and since $r_{l, j}^{\prime}<r_{l, j}$, it follows that $\mathrm{i}_{4}\left(\mathrm{~b}_{0}^{j, n}\left(X_{j}^{q} f(X)\right)\right)<\mathrm{i}_{4}(f)$ in this case. On the other hand, if $r_{l, j}^{\prime}=0$, then $j(h)<j(f)$, and again we obtain $\mathrm{i}_{4}\left(\mathrm{~b}_{0}^{j, n}\left(X_{j}^{q} f(X)\right)\right)<\mathrm{i}_{4}(f)$.

Finally, for later use we record the following consequences of the algorithm. Let $f \in \mathcal{D}_{n}$ be nonzero such that $\mathrm{h}_{n}(f)>0$, and let $\tau$ be an admissible substitution as obtained from the algorithm such that $\mathrm{h}_{n}(\phi)<\mathrm{h}_{n}(f)$ for all $\phi \in \tau f$.

Lemma 2.13. (1) Assume there are $i \in\{1, \ldots, n\}$ and $k \in \mathbb{N}$ such that $\tau=$ $\left\{p_{i, k}^{+}, p_{i, k}^{-}\right\}$. Then $i<n$, and for any integer $q \geq 0$ and $\phi \in \tau\left(X_{i}^{q} \cdot f\right)$ we have $\mathrm{h}_{n}(\phi)<\mathrm{h}_{n}(f)$.

(2) Assume there are $1 \leq i<j \leq n$ such that $\tau=b^{i, j}$. Then for every integer $q \geq 0$ we have $\mathrm{h}_{n}\left(\mathrm{~b}_{\lambda}^{i, j}\left(X_{i}^{q} \cdot f\right)\right)<\mathrm{h}_{n}(f)$ for all $\lambda \in \mathbb{R}$, and $\mathrm{h}_{n}\left(\mathrm{~b}_{\infty}^{i, j}\left(X_{j}^{q} \cdot f\right)\right)<$ $\mathrm{h}_{n}(f)$.

Proof. (1) By induction on $n$; if $n=1$, then $\mathrm{h}_{n}(g)=0$ for all nonzero $g \in \mathcal{D}_{n}$, so there is nothing to prove. We therefore assume that $n>1$ and that the lemma holds for lower values of $n$. Let $q \geq 0$ be an integer; we now distinguish several cases (depending on the stages of the algorithm). Since no power substitution is used to reduce to the case $\operatorname{ord}_{n}(f)<\infty$ or to lower $\mathrm{i}_{1}(f)$, we may assume that $\operatorname{ord}_{n}(f)<\infty$ and $\mathrm{i}_{1}(f)=0$.

Next we assume that $\mathrm{i}_{2}(f)>0$. We let $(h, g) \in \mathcal{F}_{f}\left(P_{1}\right)$ be such that $\mathrm{h}_{n-1}(\widetilde{g})=$ $\mathrm{i}_{2}(f)$, where $\widetilde{g}$ is associated to $f$ as in Step 2. Assume also that $\tau$ is obtained from Step 2 such that $\mathrm{i}_{2}(\phi)<\mathrm{i}_{2}(f)$ for all $\phi \in \tau f$. Then $\tau$ is a substitution in the variables $X^{\prime}$ such that $\mathrm{h}_{n-1}(\phi)<\mathrm{h}_{n-1}(\widetilde{g})$ for all $\phi \in \tau(\widetilde{g})$. By the inductive hypothesis $i<n-1$ and $\mathrm{h}_{n-1}(\phi)<\mathrm{h}_{n-1}(\widetilde{g})$ for all $\phi \in \tau\left(X_{i}^{q} \cdot \widetilde{g}\right)$. On the other hand, the pair $\left(X_{i}^{q} h, g\right)$ belongs to $\mathcal{F}_{X_{i}^{q} . f}$, so that $\mathrm{h}_{n}(\phi)<\mathrm{h}_{n}(f)$ for all $\phi \in \tau\left(X_{i}^{q} \cdot f\right)$.

So we may also assume that $\mathrm{i}_{2}(f)=0$. If $\mathrm{i}_{3}(f)>0$, then $i<n$ and $\mathrm{h}_{n}(\phi)<\mathrm{h}_{n}(f)$ for every $\phi \in \tau\left(X_{i}^{q} f\right)$ by Step 4 and Remark 2.9 We therefore may assume that $\mathrm{i}_{3}(f)=0$ as well. Since in this case we use a blow-up substitution to lower $\mathrm{h}_{n}(f)$, part (1) is proved.

The proof of part (2) is similar, using the corresponding observations stated in Lemmas 2.10, 2.11 and 2.12. 


\section{3. $\mathcal{C}$-SETS}

All manifolds, maps, etc., are from now on assumed to be of class $C^{\infty}$, unless otherwise indicated; in addition, submanifolds of Euclidean space are always assumed to be embedded. If $n>0$ and $x=\left(x_{1}, \ldots, x_{n}\right)$, we write $x^{\prime}=\left(x_{1}, \ldots, x_{n-1}\right)$.

Throughout the rest of this paper we fix, for every compact box $B=\left[a_{1}, b_{1}\right] \times$ $\cdots \times\left[a_{n}, b_{n}\right]$ with $a_{i}<b_{i}$ for $i=1, \ldots, n$ and $n \in \mathbb{N}$, an $\mathbb{R}$-algebra $\mathcal{C}_{B}$ of functions $f: B \longrightarrow \mathbb{R}$ such that the following hold:

(C1) $\mathcal{C}_{B}$ contains the functions $\left(x_{1}, \ldots, x_{n}\right) \mapsto x_{i}: B \longrightarrow \mathbb{R}$, and for every $f \in \mathcal{C}_{B}$, the restriction of $f$ to $\operatorname{int}(B)$ is $C^{\infty}$;

(C2) if $B^{\prime} \subseteq \mathbb{R}^{m}$ is a compact box and $g_{1}, \ldots, g_{n} \in \mathcal{C}_{B^{\prime}}$ are such that $g\left(B^{\prime}\right) \subseteq$ $B$, where $g=\left(g_{1}, \ldots, g_{n}\right)$, then for every $f \in \mathcal{C}_{B}$ the function $y \mapsto$ $f\left(g_{1}(y), \ldots, g_{n}(y)\right): B^{\prime} \longrightarrow \mathbb{R}$ belongs to $\mathcal{C}_{B^{\prime}}$

(C3) for every compact box $B^{\prime} \subseteq B$ we have $f \mid B^{\prime} \in \mathcal{C}_{B^{\prime}}$ for all $f \in \mathcal{C}_{B}$, and for every $f \in \mathcal{C}_{B}$ there is a compact box $B^{\prime} \subseteq \mathbb{R}^{n}$ and $g \in \mathcal{C}_{B^{\prime}}$ such that $B \subseteq \operatorname{int}\left(B^{\prime}\right)$ and $g \mid B=f$.

Note that (C1) and (C3) imply in particular that every $f \in \mathcal{C}_{B}$ extends to a $C^{\infty}$ function $\bar{f}: U \longrightarrow \mathbb{R}$ for some open neighborhood $U$ of $B$ (depending on $f$ ). Therefore, for each $i=1, \ldots, n$ we denote the restriction of $\partial \bar{f} / \partial x_{i}$ to $B$ by $\partial f / \partial x_{i}$. With this notation, we also assume for every compact box $B \subseteq \mathbb{R}^{n}$ that

(C4) $\partial f / \partial x_{i} \in \mathcal{C}_{B}$ for every $f \in \mathcal{C}_{B}$ and each $i=1, \ldots, n$.

Let $r=\left(r_{1}, \ldots, r_{n}\right) \in(0, \infty)^{n}$ be a polyradius, and put

$$
I_{r}:=\left(-r_{1}, r_{1}\right) \times \cdots \times\left(-r_{n}, r_{n}\right) \text { and } \overline{I_{r}}:=\operatorname{cl}\left(I_{r}\right) .
$$

(If $\epsilon>0$, we simply write $\epsilon$ for the polyradius $(\epsilon, \ldots, \epsilon)$.) From now on, we write $\mathcal{C}_{n, r}:=\mathcal{C}_{\overline{I_{r}}}$. We denote by $\mathcal{C}_{n}$ the collection of all germs at the origin of the functions in $\bigcup_{r \in(0, \infty)^{n}} \mathcal{C}_{n, r}$. Note that each $\mathcal{C}_{n}$ is an $\mathbb{R}$-algebra with respect to the usual addition and multiplication of germs at 0 . Finally, we let ${ }^{\wedge}: \mathcal{C}_{n} \longrightarrow \mathbb{R} \llbracket X \rrbracket$ be the map that sends each $f \in \mathcal{C}_{n}$ to its Taylor series $\widehat{f}$ at the origin, and we denote the image of $\widehat{ }{ }^{-}$in $\mathbb{R} \llbracket X \rrbracket$ by $\widehat{\mathcal{C}_{n}}$.

In addition to (C1)-(C4) above, we make the following assumptions: for all $n \geq 1$, we have

(C5) $\widehat{\wedge}: \mathcal{C}_{n} \longrightarrow \widehat{\mathcal{C}_{n}}$ is an $\mathbb{R}$-algebra isomorphism (quasianalyticity);

(C6) if $n>1$ and $f \in \mathcal{C}_{n}$ is such that $f(0)=0$ and $\left(\partial f / \partial x_{n}\right)(0) \neq 0$, there is an $\alpha \in \mathcal{C}_{n-1}$ with $\alpha(0)=0$ such that $f\left(x^{\prime}, \alpha\left(x^{\prime}\right)\right)=0$;

(C7) if $f \in \mathcal{C}_{n}$ and $i \leq n$ are such that $\widehat{f}(X)=X_{i} G(X)$ for some $G \in \mathbb{R} \llbracket X \rrbracket$, then $f=x_{i} g$ for some $g \in \mathcal{C}_{n}$ such that $G=\widehat{g}$.

It follows from these assumptions that the collection $\widehat{\mathcal{C}}:=\left(\widehat{\mathcal{C}_{n}}\right)_{n \in \mathbb{N}}$ satisfies $(\mathrm{Q} 1)-$ (Q5).

Examples 3.1. (1) Let $M=\left(M_{0}, M_{1}, \ldots\right)$ be a strongly log-convex sequence satisfying QA. Then, as discussed in the introduction and the appendix, the classes $\mathcal{C}_{B}(M)$, with $B \subseteq \mathbb{R}^{n}$ a compact box and $n \in \mathbb{N}$, satisfy (C1)-(C7).

(2) Let $\mathcal{R}$ be a polynomially bounded o-minimal expansion of the real field. For every compact box $B \subseteq \mathbb{R}^{n}$, let $\mathcal{C}_{B}$ be the collection of all functions on $B$ that are the restriction to $B$ of some definable $C^{\infty}$ function defined on an open neighborhood of $B$. Then by [16], the classes $\mathcal{C}_{B}$ satisfy $(\mathrm{C} 1)-(\mathrm{C} 7)$. 
Definition 3.2. A set $A \subseteq \mathbb{R}^{n}$ is called a basic $\mathcal{C}$-set if there are $r \in(0, \infty)^{n}$ and $f, g_{1}, \ldots, g_{k} \in \mathcal{C}_{n, r}$ such that

$$
A=\left\{x \in I_{r}: f(x)=0, g_{1}(x)>0, \ldots, g_{k}(x)>0\right\} .
$$

A finite union of basic $\mathcal{C}$-sets is called a $\mathcal{C}$-set. We call $M \subseteq \mathbb{R}^{n}$ a $\mathcal{C}$-manifold if there is an $r \in(0, \infty)^{n}$ such that

(1) $M$ is a basic $\mathcal{C}$-set contained in $I_{r}$, and

(2) there are $f_{1}, \ldots, f_{k} \in \mathcal{C}_{n, r}$ such that $M$ is a submanifold of $I_{r}$ of dimension $n-k$ on which $f_{1}, \ldots, f_{k}$ vanish identically, and the gradients $\nabla f_{1}(z), \ldots, \nabla f_{k}(z)$ are linearly independent at each $z \in M$.

As in [8], we say that a set $S \subseteq \mathbb{R}^{n}$ has dimension if $S$ is a countable union of manifolds of class $C^{1}$, and in that case we put

$$
\operatorname{dim}(S):= \begin{cases}\max \left\{\operatorname{dim}(M): M \subseteq S \text { is a } C^{1} \text { manifold }\right\} & \text { if } S \neq \emptyset, \\ -\infty & \text { otherwise. }\end{cases}
$$

Remarks 3.3. (1) If $S=\bigcup_{i \in \mathbb{N}} S_{i}$ and each $S_{i}$ has dimension, then $S$ has dimension and $\operatorname{dim}(S)=\max \left\{\operatorname{dim}\left(S_{i}\right): i \in \mathbb{N}\right\}$. (This follows by an elementary Baire category argument.)

(2) If $M$ is a manifold, then $\operatorname{dim}(M)$ in the sense of the previous definition agrees with the usual manifold dimension.

Given $m \leq n$, we let $\Pi_{m}^{n}: \mathbb{R}^{n} \longrightarrow \mathbb{R}^{m}$ be the projection map given by $\Pi_{m}^{n}\left(x_{1}, \ldots, x_{n}\right)=\left(x_{1}, \ldots, x_{m}\right)$. More generally, given an injective $\lambda:\{1, \ldots, m\} \rightarrow$ $\{1, \ldots, n\}$, we let $\Pi_{\lambda}^{n}: \mathbb{R}^{n} \longrightarrow \mathbb{R}^{m}$ be the projection $\Pi_{\lambda}^{n}(x):=\left(x_{\lambda(1)}, \ldots, x_{\lambda(m)}\right)$. We will simply write $\Pi_{m}$ for $\Pi_{m}^{n}$ and $\Pi_{\lambda}$ for $\Pi_{\lambda}^{n}$ if $n$ is clear from context.

For $r \in(0, \infty)^{n}, f=\left(f_{1}, \ldots, f_{\mu}\right) \in\left(\mathcal{C}_{n, r}\right)^{\mu}, S \subseteq I_{r}$ and a sign condition $\sigma \in$ $\{-1,0,1\}^{\mu}$ we put

$$
B_{S}(f, \sigma):=\left\{x \in S: \operatorname{sgn} f_{1}(x)=\sigma_{1}, \ldots, \operatorname{sgn} f_{\mu}(x)=\sigma_{\mu}\right\} .
$$

Definition 3.4. Let $r \in(0, \infty)^{n}$. A set $M \subseteq I_{r}$ is $\mathcal{C}$-trivial if one of the following holds:

(1) $M=B_{I_{r}}\left(\left(x_{1}, \ldots, x_{n}\right), \sigma\right)$ for some sign condition $\sigma \in\{-1,0,1\}^{n}$, or

(2) there are a permutation $\lambda$ of $\{1, \ldots, n\}$, a $\mathcal{C}$-trivial $N \subseteq I_{s}$ and a $g \in$ $\mathcal{C}_{n-1, s}$, where $s=\left(r_{\lambda(1)}, \ldots, r_{\lambda(n-1)}\right)$, such that $g\left(I_{s}\right) \subseteq\left(-r_{\lambda(n)}, r_{\lambda(n)}\right)$ and $\Pi_{\lambda}(M)=\operatorname{gr}(g \mid N)$.

Remarks 3.5. Let $r \in(0, \infty)^{n}$ and $M \subseteq I_{r}$ be $\mathcal{C}$-trivial. Using $(\mathrm{C} 1)-(\mathrm{C} 4)$, the following observations are obtained by induction on $n$ :

(1) $M$ is a bounded and connected $\mathcal{C}$-manifold, and fr $M:=\operatorname{cl}(M) \backslash M$ is a $\mathcal{C}$-set, has dimension, and $\operatorname{dim}(\operatorname{fr} M)<\operatorname{dim}(M)$.

(2) For every $z \in$ fr $M$, there are $\delta>0$ and $\gamma=\left(\gamma_{1}, \ldots, \gamma_{n}\right) \in\left(\mathcal{C}_{1, \delta}\right)^{n}$ such that $\gamma(t) \in M$ for every $t \in(0, \delta)$ and $\gamma(0)=z$.

Definition 3.6. Let $r \in(0, \infty)^{n}$ and $f=\left(f_{1}, \ldots, f_{\mu}\right) \in \mathcal{C}_{n, r}^{\mu}$. We denote by $\mathbb{R}\langle f\rangle$ the expansion of the real field generated by the functions

$$
x \mapsto \begin{cases}\frac{\partial^{\alpha} f_{\nu}}{\partial x^{\alpha}}(x) & \text { if } x \in \overline{I_{r}}, \\ 0 & \text { otherwise, }\end{cases}
$$


where $\nu=1, \ldots, \mu$ and $\alpha \in \mathbb{N}^{n}$. Also let $l, m \in \mathbb{N}, s \in(0, \infty)^{m}$ and $A_{i} \subseteq I_{s}$ a basic $\mathcal{C}$-set for $i=1, \ldots, l$, say

$$
A_{i}=\left\{y \in I_{s}: h_{i}(y)=0, g_{i, 1}(y)>0, \ldots, g_{i, k(i)}(y)>0\right\}
$$

with $h_{i}, g_{i, j} \in \mathcal{C}_{m, s}$ for $i=1, \ldots, l$ and $j=1, \ldots, k(i)$. We say that the $\mathcal{C}$-set $A:=\bigcup_{i=1}^{l} A_{i}$ is $\Delta$-definable from $f$ if for every $\beta \in \mathbb{N}^{m}$, the functions $\partial^{\beta} h_{i} / \partial y^{\beta}$ and $\partial^{\beta} g_{i, j} / \partial y^{\beta}$, for all $i \in\{1, \ldots, l\}$ and all $j \in\{1, \ldots, k(i)\}$, are quantifier-free definable in $\mathbb{R}\langle f\rangle$.

Example 3.7. The previous definition will be relevant in situations similar to the following. Let $r \in(0, \infty)^{n}$ and $f \in \mathcal{C}_{n, r}$ be such that $f(0)=0$ and $\partial f / \partial x_{n}(0) \neq 0$. Then there is an $s \in(0, \infty)^{n}$ and an $\alpha \in \mathcal{C}_{n-1, s^{\prime}}$, where $s^{\prime}=\left(s_{1}, \ldots, s_{n-1}\right)$, such that $\alpha(0)=0$ and for all $x \in I_{s}$,

$$
f(x)=0 \quad \text { if and only if } \quad x_{n}=\alpha\left(x^{\prime}\right) .
$$

It follows from Remark 2.4 $(3)$ that for any $\epsilon>0$, after suitably shrinking $s$, the set $\left\{(x, t) \in I_{(s, \epsilon)}: t=x_{n}+\alpha\left(x^{\prime}\right)\right\}$ is $\Delta$-definable from $f$. If, in addition, $B \subseteq I_{s}$ is a $\mathcal{C}$-set that is $\Delta$-definable from $f$, then the set

$$
\left\{(x, t) \in I_{(s, \epsilon)}: x \in B \text { and } t=x_{n}+\alpha\left(x^{\prime}\right)\right\}
$$

is also $\Delta$-definable from $f$.

Proposition 3.8. Let $r \in(0, \infty)^{n}$ and $f \in\left(\mathcal{C}_{n, r}\right)^{\mu}$. Then there is a neighborhood $W \subseteq I_{r}$ of 0 with the following property:

(*) for every sign condition $\sigma \in\{-1,0,1\}^{\mu}$ there is an $l \in \mathbb{N}$, and for each $k=$ $1, \ldots, l$ there are $n_{k} \geq n, r_{k} \in(0, \infty)^{n_{k}}$ and $\mathcal{C}$-trivial manifolds $N_{k} \subseteq I_{r_{k}}$ that are $\Delta$-definable from $f$, such that

$$
B_{W}(f, \sigma)=\Pi_{n}\left(N_{1}\right) \cup \cdots \cup \Pi_{n}\left(N_{l}\right)
$$

and for each $k$, the set $\Pi_{n}\left(N_{k}\right)$ is a manifold and $\Pi_{n} \mid N_{k}: N_{k} \longrightarrow \Pi_{n}\left(N_{k}\right)$ is a diffeomorphism.

Proof. We may clearly assume that $f_{\nu} \neq 0$ for each $\nu=1, \ldots, \mu$. Let $g:=f_{1} \cdots f_{\mu}$; then $g \neq 0$, so by the quasianalyticity of $\mathcal{C}_{n}$ we have $\widehat{g} \neq 0$.

We proceed by induction on the pair $\left(n, \mathrm{~h}_{n}(\widehat{g})\right)$, where $\mathrm{h}_{n}$ is the function measuring nonnormality of power series as introduced in Section 2 If $\mathrm{h}_{n}(\widehat{g})=0$, the proposition follows from $(\mathrm{C} 7)$, so we assume that $n>1, \mathrm{~h}_{n}(\widehat{g})>0$ and the proposition holds for lower values of $\left(n, \mathrm{~h}_{n}(\widehat{g})\right)$. By Theorem 2.5, there is an admissible substitution $\tau$ such that $\mathrm{h}_{n}(\phi)<\mathrm{h}_{n}(\widehat{g})$ for all $\phi \in \tau \widehat{g}$.

Note by (C5) that each $\xi \in \tau$ corresponds to a unique germ $h_{\xi} \in\left(\mathcal{C}_{n}\right)^{n}$, and that $\widehat{h \circ h_{\xi}}=\xi \widehat{h}$ for all $h \in \mathcal{C}_{n}$. Thus to simplify notation below, we do not distinguish any longer between $h \in \mathcal{C}_{n}$ and $\widehat{h} \in \widehat{\mathcal{C}}_{n}$, or between $\xi$ and $h_{\xi}$.

Below $\mathcal{C}_{n, r}$ is viewed as a subset of $\mathcal{C}_{n}$ by identifying each $g \in \mathcal{C}_{n, r}$ with its germ in $\mathcal{C}_{n}$. Correspondingly, given $g=\left(g_{1}, \ldots, g_{k}\right) \in\left(\mathcal{C}_{n}\right)^{k}$, we say that $r \in(0, \infty)^{n}$ is $g$-small if $g_{i} \in \mathcal{C}_{n, r}$ for $i=1, \ldots, k$.

Case 1. $\tau=\left\{t_{\alpha}\right\}$ for some $\alpha \in \mathcal{C}_{i-1}$ with $1<i \leq n$ and $\alpha(0)=0$. Let $s \in(0, \infty)^{n}$ be both $\left(t_{\alpha} f\right)$-small and $t_{\alpha}$-small and such that $t_{\alpha}\left(I_{s}\right) \subseteq I_{r}$. By the inductive hypothesis, there is a neighborhood $V \subseteq I_{s}$ of 0 such that $(*)$ holds with $t_{\alpha} f, s$ and $V$ in place of $f, r$ and $W$. Then $W=t_{\alpha}(V)$ is a neighborhood of 0 , and we claim that $(*)$ holds with this $W$. 
To see this, we let $\sigma \in\{-1,0,1\}^{\mu}$. Let $M_{k} \subseteq \mathbb{R}^{m_{k}}$ for $k=1, \ldots, p$ be the $\mathcal{C}$ trivial manifolds obtained for this $\sigma$ from the inductive hypothesis applied to $t_{\alpha} f$; in particular, each $M_{k}$ is $\Delta$-definable from $t_{\alpha} f$. By Remark 2.4(3), it follows that each $M_{k}$ is $\Delta$-definable from $f$. For each $k$ we put

$$
N_{k}:=\left\{\left(x_{<i}, t, x_{>i}, x_{i}\right): x \in M_{k} \text { and } t=x_{i}+\alpha\left(x_{<i}\right)\right\},
$$

where $x_{<i}:=\left(x_{1}, \ldots, x_{i-1}\right)$ and $x_{>i}:=\left(x_{i+1}, \ldots, x_{m_{k}}\right)$. Then by Example 3.7] each $N_{k}$ is a $\mathcal{C}$-trivial manifold that is $\Delta$-definable from $f$, and $B_{W}(f, \sigma)=\bigcup_{k=1}^{p} \Pi_{n}\left(N_{k}\right)$. Moreover, since $t_{\alpha} \mid I_{s}: I_{s} \longrightarrow t_{\alpha}\left(I_{s}\right)$ is a diffeomorphism, it follows that $\Pi_{n}\left(N_{k}\right)=$ $t_{\alpha}\left(\Pi_{n}\left(M_{k}\right)\right)$ is a manifold and $\Pi_{n} \mid N_{k}: N_{k} \longrightarrow \Pi_{n}\left(N_{k}\right)$ a diffeomorphism, as required.

Case 2. $\tau=\left\{l_{i, c}\right\}$ for some $1<i \leq n$ and $c \in \mathbb{R}^{i-1}$. In this case, the proof is similar to the proof of Case 1 . Here and in Cases 3 and 4 below, the $\Delta$-definability of the manifolds in question follows from the inductive hypothesis because $\tau$ is semialgebraic.

Case 3. There are $i \in\{1, \ldots, n\}$ and $d>1$ such that $\tau=\left\{p_{i, d}^{+}, p_{i, d}^{-}\right\}$; below we write $p^{+}$and $p^{-}$in place of $p_{i, d}^{+}$and $p_{i, d}^{-}$. Then by Lemma 2.13(1), $i<n$ and $\mathrm{h}_{n}\left(p^{+}\left(X_{i} \cdot g\right)\right)<\mathrm{h}_{n}(g)$ and $\mathrm{h}_{n}\left(p^{-}\left(X_{i} \cdot g\right)\right)<\mathrm{h}_{n}(g)$. On the other hand, since for any set $S \subseteq \mathbb{R}^{n}$ and any $\sigma \in\{-1,0,1\}^{\mu}$,

$$
B_{S}(f, \sigma)=\bigcup_{\chi \in\{-1,0,1\}} B_{S}\left(\left(f_{1}, \ldots, f_{\mu}, X_{i}\right), \sigma^{\wedge} \chi\right),
$$

where $\sigma^{\wedge} \chi(i)=\sigma(i)$ if $i \leq \mu$ and $\sigma^{\wedge} \chi(\mu+1)=\chi$ for each $\chi$, we may as well assume that $f_{\mu}(X)=X_{i}$. Below we write $\mathbb{R}_{i, *}^{n}=\left\{x \in \mathbb{R}^{n}: x_{i} * 0\right\}$ for $* \in\{<, 0,>, \neq\}$.

Let $s \in(0, \infty)^{n}$ be both $\left(p^{+} f\right)$-small and $\left(p^{-} f\right)$-small such that $p^{+}\left(I_{s}\right) \subseteq I_{r}$ and $p^{-}\left(I_{s}\right) \subseteq I_{r}$. By the inductive hypothesis, there are neighborhoods $V^{+}, V^{-} \subseteq I_{s}$ of 0 such that $(*)$ holds with $p^{*} f, s$ and $V^{*}$ in place of $f, r$ and $W$ (where $* \in\{+,-\}$ ). Also by the inductive hypothesis, $(*)$ holds with $f \mid \mathbb{R}_{i, 0}^{n}, s$ and a neighborhood $V^{0} \subseteq$ $\mathbb{R}_{i, 0}^{n}$ of 0 in place of $f, r$ and $W$. Then $W_{1}:=p^{+}\left(V^{+} \cap \mathbb{R}_{i,>}^{n}\right) \cup V^{0} \cup p^{-}\left(V^{-} \cap \mathbb{R}_{i,<}^{n}\right)$ and $W_{2}:=p^{+}\left(V^{+} \backslash \mathbb{R}_{i, \neq}^{n}\right) \cup V_{0}$ are neighborhoods of 0 , and we claim that $(*)$ holds with $W_{1}$ (resp. $W_{2}$ ) in place of $W$ if $d$ is even (resp. odd).

To see this, we let $\sigma \in\{-1,0,1\}^{\mu}$; if $\sigma(\mu)=0$, then $B_{W}(f, \sigma)=B_{V^{0}}(f, \sigma)$ and the claim follows. Assume now that $\sigma(\mu)=1$ and $d$ is even; the proof for $\sigma(\mu)=-1$ or $d$ odd is similar. Then $B_{V^{+}}\left(p^{+} f, \sigma\right) \subseteq \mathbb{R}_{i, \neq}^{n}$, so because $\mathcal{C}$-trivial manifolds are connected and $\mathbb{R}_{i, \neq}^{n}$ has two connected components, (*) also holds with $p^{+} f, s$ and $V^{+} \cap \mathbb{R}_{i,>}^{n}$ and with $p^{-} f, s$ and $V^{-} \cap \mathbb{R}_{i,<}^{n}$ in place of $f, r$ and $W$. Since $p^{+} \in \mathcal{C}_{n}$ and $p^{+} \mid \mathbb{R}_{i,>}^{n}$ is a diffeomorphism of $\mathbb{R}_{i,>}^{n}$ onto itself and, similarly, $p^{-} \in \mathcal{C}_{n}$ and $p^{-} \mid \mathbb{R}_{i,<}^{n}$ is a diffeomorphism of $\mathbb{R}_{i,<}^{n}$ onto itself, we finish with a similar argument as in the proof of Case 1.

Case 4. $\tau=\mathrm{b}^{i, j}$ for some $1 \leq i<j \leq n$; below we write $\mathrm{b}$ instead of $\mathrm{b}^{i, j}$ and put $Z:=\left\{x_{i}=x_{j}=0\right\}$. (Note that $Z$ is the center of the blowing-up whose charts are represented by b.)

First, we fix an arbitrary $\lambda \in \mathbb{R}$ and let $s_{\lambda} \in(0, \infty)^{n}$ be $\left(\mathrm{b}_{\lambda} f\right)$-small such that $\mathrm{b}_{\lambda}\left(I_{s_{\lambda}}\right) \subseteq I_{r}$. Then by Lemma 2.13] 2$), \mathrm{h}_{n}\left(\mathrm{~b}_{\lambda}\left(X_{i} \cdot g\right)\right)<\mathrm{h}_{n}(g)$. Thus by the inductive hypothesis, there is a neighborhood $V_{\lambda} \subseteq I_{\delta_{\lambda}}$ of 0 such that $(*)$ holds with $\left(\mathrm{b}_{\lambda} f, \mathrm{~b}_{\lambda}\left(X_{i}\right)\right), s_{\lambda}$ and $V_{\lambda}$ in place of $f, r$ and $W$. Since $\mathrm{b}_{\lambda}\left(X_{i}\right)=X_{i}, \mathrm{~b}_{\lambda}$ is 
continuous and $\mathrm{b}_{\lambda} \mid \mathbb{R}_{i, \neq}^{n}$ is a diffeomorphism of $\mathbb{R}_{i, \neq}^{n}$ onto itself, it follows by a similar argument as in Case 3 that $(*)$ holds with $f, s_{\lambda}$ and $W_{\lambda}:=\mathrm{b}_{\lambda}\left(V_{\lambda}\right) \backslash \mathbb{R}_{i, 0}^{n}=\mathrm{b}_{\lambda}\left(V_{\lambda}\right) \backslash Z$ in place of $f, r$ and $W$. (Of course, $W_{\lambda}$ is not a neighborhood of 0 .)

Next, let $s_{\infty} \in(0, \infty)^{n}$ be $\left(\mathrm{b}_{\infty} f\right)$-small such that $\mathrm{b}_{\infty}\left(I_{s_{\infty}}\right) \subseteq I_{r}$. Again by Lemma 2.13] $(2), \mathrm{h}_{n}\left(\mathrm{~b}_{\infty}\left(X_{j} \cdot g\right)\right)<\mathrm{h}_{n}(g)$. Hence, just as for $\lambda \in \mathbb{R}$, there is a neighborhood $V_{\infty} \subseteq I_{s_{\infty}}$ of 0 such that $(*)$ holds with $f, s_{\infty}$ and $W_{\infty}:=\mathrm{b}_{\infty}\left(V_{\infty}\right) \backslash Z$ in place of $f, r$ and $W$.

Finally, by the inductive hypothesis there is a neighborhood $W^{\prime} \subseteq Z$ of 0 such that (*) holds with $f \mid Z$ and $W^{\prime}$ in place of $f$ and $W$. Now note that there is a finite set $S \subseteq \mathbb{Z}$ such that $W:=W^{\prime} \cup W_{\lambda_{0}} \cup W_{\lambda_{\infty}} \cup \bigcup_{s \in S} W_{\lambda_{s}}$ is a neighourhood of 0 .

\section{4. $\mathcal{C}$-Semianalytic Sets}

Definition 4.1. Let $A \subseteq \mathbb{R}^{n}$. The set $A$ is called $\mathcal{C}$-semianalytic at $a \in \mathbb{R}^{n}$ if there is an $r \in(0, \infty)^{n}$ such that $(A-a) \cap I_{r}$ is a $\mathcal{C}$-set. $A$ is $\mathcal{C}$-semianalytic if $A$ is $\mathcal{C}$-semianalytic at every point $a \in \mathbb{R}^{n}$. If moreover $A$ is a manifold, then we call $A$ a $\mathcal{C}$-semianalytic manifold.

Remarks. (1) If $A, B \subseteq \mathbb{R}^{n}$ are $\mathcal{C}$-semianalytic at $a$, then so are $A \cup B, A \cap B$ and $A \backslash B$.

(2) If $A \subseteq \mathbb{R}^{n}$ is $\mathcal{C}$-semianalytic, then by $(\mathrm{C} 2)$ the set $E_{\lambda}(A)$ is $\mathcal{C}$-semianalytic for each $\lambda \in(\mathbb{R} \backslash\{0\})^{n}$, where $E_{\lambda}: \mathbb{R}^{n} \longrightarrow \mathbb{R}^{n}$ is given by $E_{\lambda}(x)=\left(\lambda_{1} x_{1}, \ldots, \lambda_{n} x_{n}\right)$.

(3) By $(\mathrm{C} 1)$ and $(\mathrm{C} 2)$, every $\mathcal{C}$-set is $\mathcal{C}$-semianalytic.

To obtain the strengthening of Theorem 1 mentioned in the introduction, we need the following.

Definition 4.2. Let $A \subseteq \mathbb{R}^{n}$ be bounded and $\mathcal{C}$-semianalytic. Then there are $a_{1}, \ldots, a_{l} \in \mathbb{R}^{n}$ and $r_{1}, \ldots, r_{l} \in(0, \infty)^{n}$ such that $A \subseteq\left(I_{r_{1}}+a_{1}\right) \cup \cdots \cup\left(I_{r_{l}}+a_{l}\right)$ and $\left(A-a_{i}\right) \cap I_{r_{i}}$ is a $\mathcal{C}$-set for each $i=1, \ldots, l$. Also let $s_{j} \in(0, \infty)^{m_{j}}$ and $f_{j} \in \mathcal{C}_{m_{j}, s_{j}}^{\mu_{j}}$, for $j=1, \ldots, k$. We say that $A$ is $\Delta$-definable from $\left\{f_{1}, \ldots, f_{k}\right\}$ if for each $i \in\{1, \ldots, l\}$, there is a $j \in\{1, \ldots, k\}$ such that $\left(A-a_{i}\right) \cap I_{r_{i}}$ is $\Delta$-definable from $f_{j}$.

Assume in addition that for $i \in\{1, \ldots, l\}$,

$$
\left(A-a_{i}\right) \cap I_{r_{i}}=\bigcup_{j=1}^{p(i)}\left\{x \in I_{r_{i}}: h_{i, j}(x)=0, g_{i, j, 1}(x)>0, \ldots, g_{i, j, k(i, j)}>0\right\}
$$

with $h_{i, j}, g_{i, j, 1}, \ldots, g_{i, j, k(i, j)} \in \mathcal{C}_{n, r_{i}}$, and let $B \subseteq \mathbb{R}^{p}$ be $\mathcal{C}$-semianalytic. Then $B$ is called $\Delta$-definable from $A$ if $B$ is $\Delta$-definable from the collection

$$
\left\{\left(h_{i, 1}, g_{i, 1,1}, \ldots, g_{i, p(i), k(i, p(i))}\right): i=1, \ldots, l\right\} \text {. }
$$

Remark 4.3. Let $A, B$ and $C$ be $\mathcal{C}$-semianalytic, such that $B$ is $\Delta$-definable from $A$ and $C$ is $\Delta$-definable from $B$. Then $C$ is $\Delta$-definable from $A$.

A $\mathcal{C}$-semianalytic manifold $M \subseteq \mathbb{R}^{n}$ is called trivial if $M=N+a$ for some $\mathcal{C}$-trivial manifold $N \subseteq \mathbb{R}^{n}$ and some $a \in \mathbb{R}^{n}$. (Thus any trivial $\mathcal{C}$-semianalytic manifold is bounded and connected.) 
Corollary 4.4. Let $A \subseteq \mathbb{R}^{n}$ be bounded and $\mathcal{C}$-semianalytic. Then there are $n_{i} \geq n$ and trivial $\mathcal{C}$-semianalytic manifolds $N_{i} \subseteq \mathbb{R}^{n_{i}}$ for $i=1, \ldots, k$, each $\Delta$-definable from $A$, such that

$$
A=\Pi_{n}\left(N_{1}\right) \cup \cdots \cup \Pi_{n}\left(N_{k}\right),
$$

and for each $i$, the set $\Pi_{n}\left(N_{i}\right)$ is a manifold and $\Pi_{n} \mid N_{i}: N_{i} \longrightarrow \Pi_{n}\left(N_{i}\right)$ is a diffeomorphism. In particular, A has dimension.

Proof. By the definition of "C $\mathrm{C}$-semianalytic" and Proposition 3.8, the corollary holds locally at each point of $\mathbb{R}^{n}$, and hence the boundedness of $A$ implies that it holds globally.

Let $M \subseteq \mathbb{R}^{n}$ be a $\mathcal{C}$-manifold of dimension $m \leq n$ with associated $r \in(0, \infty)^{n}$ and $f_{1}, \ldots, f_{n-m} \in \mathcal{C}_{n, r}$ as in Definition 3.2 (with $n-m$ in place of $k$ ). Given a strictly increasing $\iota:\{1, \ldots, m\} \longrightarrow\{1, \ldots, n\}$, we put

$$
M_{\iota}:=\left\{x \in M: \Pi_{\iota} \mid T_{x} M \text { has rank } m\right\} .
$$

Then $M_{\iota}$ is an open subset of $M$ and is in fact a $\mathcal{C}$-manifold (with same associated $r$ and $\left.f_{1}, \ldots, f_{n-m}\right)$. Moreover, the assumption on $f_{1}, \ldots, f_{n-m}$ implies that

$$
M=\bigcup\left\{M_{\iota}: \iota:\{1, \ldots, m\} \longrightarrow\{1, \ldots, n\} \text { is strictly increasing }\right\} .
$$

Let $k \leq m$ and $\iota:\{1, \ldots, m\} \longrightarrow\{1, \ldots, n\}$ be strictly increasing. We let $m(k) \in\{0, \ldots, m\}$ be maximal such that $\iota(m(k)) \leq k$ and put $\iota_{k}:=\iota \mid\{1, \ldots, m(k)\}$ and $\iota^{k}:=\iota \mid\{m(k)+1, \ldots, m\}$.

Assume now that $M=M_{\iota}$ and that $\Pi_{k} \mid M$ has constant rank $m(k)$. Then by the rank theorem, each fiber $M_{a}:=\Pi_{k}^{-1}(a) \cap M$ over $a \in \mathbb{R}^{k}$ is either empty or a $\mathcal{C}$-manifold of dimension $m-m(k)$, and $\Pi_{\iota^{k}} \mid M_{a}$ is an immersion. (To see the latter, note that for $x \in M_{a}$ the tangent space $T_{x} M_{a}$ is a subspace of $T_{x} M$ of dimension $e:=m-m(k)$ such that $\Pi_{k}\left(T_{x} M_{a}\right)=\{0\}$. Let $v_{1}, \ldots, v_{e}$ be a basis of $T_{x} M_{a}$; then $\Pi_{\iota}\left(v_{1}\right), \ldots, \Pi_{\iota}\left(v_{e}\right)$ are linearly independent in $\mathbb{R}^{m}$ and $\Pi_{\iota_{k}}\left(v_{1}\right)=\cdots=\Pi_{\iota_{k}}\left(v_{e}\right)=0$. Hence $\Pi_{\iota^{k}}\left(v_{1}\right), \ldots, \Pi_{\iota^{k}}\left(v_{e}\right)$ are linearly independent in $\mathbb{R}^{e}$.) It follows that if $C$ is a connected component of $M_{a}$, then $\Pi_{\iota^{k}}(C)$ is open in $\mathbb{R}^{e}$, which implies (since $C$ is bounded) that fr $C \neq \emptyset$ if $e \geq 1$.

Lemma 4.5 (Fiber Cutting Lemma). Let $n \geq m>k \geq 0$. Let $M \subseteq \mathbb{R}^{n}$ be a $\mathcal{C}$ manifold of dimension $m$. Assume that $M=M_{\iota}$ for some fixed strictly increasing $\iota:\{1, \ldots, m\} \longrightarrow\{1, \ldots, n\}$, and that $\Pi_{k} \mid M$ has constant rank $m(k)$. Then there is a $\mathcal{C}$-set $A \subseteq M, \Delta$-definable from $M$, such that $\operatorname{dim}(A)<m$ and $\Pi_{k}(M)=\Pi_{k}(A)$.

Proof. Note that $k<m$ implies $m(k)<m$. Let $r \in(0, \infty)^{n}$ be associated to $M$ as in Definition 3.2 (with $n-m$ in place of $k$ there), and let $f, g_{1}, \ldots, g_{k} \in \mathcal{C}_{n, r}$ be such that

$$
M=\left\{x \in I_{r}: f(x)=0, g_{1}(x)>0, \ldots, g_{k}(x)>0\right\} .
$$

Let $g$ be the product of all $g_{j}, j=1, \ldots, k$, and all $\left(x_{i}-r_{i}\right) \mid I_{r}$ and $\left(r_{i}-x_{i}\right) \mid I_{r}$, $i=1, \ldots, n$. Then $g \in \mathcal{C}_{n, r}$, and $g$ is strictly positive on all of $M$ and identically zero on fr $M$.

Next, by the paragraph preceding this lemma, for each $a \in \Pi_{k}(M)$ the fiber $M_{a}$ is a manifold of dimension $m-m(k)>0$, and fr $C \neq \emptyset$ for each connected component $C$ of $M_{a}$. Therefore, $g \mid M_{a}$ has critical points on each connected component of $M_{a}$, since $g$ is positive on $M_{a}$ and vanishes identically on fr $M_{a}$. Moreover, since $M_{a}$ is a $\mathcal{C}$-manifold, it follows from $(\mathrm{C} 5)$ and (C6) (by similar arguments as in the situation 
where $g$ is analytic) that the set of critical points of $g \mid M_{a}$ has empty interior in $M_{a}$. Let

$$
A=\left\{x \in M: x \text { is a critical point of } g \mid M_{a}, a=\Pi_{k}(x)\right\} .
$$

Then $\Pi_{k}(A)=\Pi_{k}(M)$ and $A$ is a $\mathcal{C}$-set, so $A$ has dimension by Corollary 4.4 Moreover, it follows from the definition of $g$ that $A$ is $\Delta$-definable from $M$. Since $A$ has empty interior in $M$, we have $\operatorname{dim}(A)<\operatorname{dim}(M)$.

For any manifold $M \subseteq \mathbb{R}^{n}$ of dimension $m$ and $k \leq m$, we define

$$
r(M):=\max \left\{\operatorname{rk}\left(\left.\Pi_{k}\right|_{T_{x} M}\right): x \in M\right\} \leq m .
$$

(We do not explicitly indicate the dependence of $r(M)$ on $k, m$ and $n$.)

Lemma 4.6. Let $M \subseteq \mathbb{R}^{n}$ be a $\mathcal{C}$-manifold of dimension $m$ and $k \leq m$. Then

$(*)$ there are trivial $\mathcal{C}$-semianalytic manifolds $N_{i} \subseteq \mathbb{R}^{n_{i}}, \Delta$-definable from $M$ and satisfying $\operatorname{dim}\left(N_{i}\right) \leq k$ and $n_{i} \geq n$ for $i=1, \ldots, K$, and there are bounded $\mathcal{C}$-semianalytic sets $A_{j} \subseteq \mathbb{R}^{p_{j}}, \Delta$-definable from $M$ and satisfying $\operatorname{dim}\left(A_{j}\right)<m$ and $p_{j} \geq n$ for $j=1, \ldots, L$, such that

$$
\Pi_{k}(M)=\Pi_{k}\left(N_{1}\right) \cup \cdots \cup \Pi_{k}\left(N_{K}\right) \cup \Pi_{k}\left(A_{1}\right) \cup \cdots \cup \Pi_{k}\left(A_{L}\right),
$$

and for each $i$ there is a strictly increasing $\iota:\left\{1, \ldots, \operatorname{dim}\left(N_{i}\right)\right\} \rightarrow\{1, \ldots, k\}$ such that $\Pi_{\iota} \mid N_{i}: N_{i} \longrightarrow \mathbb{R}^{\operatorname{dim}\left(N_{i}\right)}$ is an immersion.

Proof. We prove this lemma by induction on $r(M)$ simultaneously for all $k, m$ and $n$. The initial case $r(M)=0$ is trivial (since then $\Pi_{k}$ is constant on each component of $M)$, so we assume $r(M)>0$ and that the lemma holds for lower values of $r(M)$. First, we claim that it suffices to prove $(*)$ with $\widetilde{M}:=M \backslash \bigcup \mathcal{M}$ in place of $M$, where

$$
\mathcal{M}:=\left\{M_{\iota}: \iota:\{1, \ldots, m\} \longrightarrow\{1, \ldots, n\} \text { strictly increasing and } m(k)=r(M)\right\} .
$$

To see this, we let $M_{\iota} \in \mathcal{M}$ be such that $M_{\iota} \neq \emptyset$, and we show that the lemma holds with $M_{\iota}$ in place of $M$. If $k=m$, this is trivial. On the other hand, since $M_{\iota}$ is open in $M$, we have for every $x \in M_{\iota}$ that

$$
r(M)=m(k) \leq \operatorname{rk}\left(\Pi_{k} \mid T_{x} M_{\iota}\right) \leq r(M) .
$$

Hence if $k<m$, the lemma with $M_{\iota}$ in place of $M$ follows from the fiber cutting lemma, which together with Remark 4.3 proves the claim.

Next, note that for every $x \in \widetilde{M}$ the rank of $\Pi_{k} \mid T_{x} M$ is less than $r(M)$. Since $\widetilde{M}$ is clearly a bounded $\mathcal{C}$-set, we may apply Corollary 4.4 with $\widetilde{M}$ in place of $A$. Denote by $M_{\nu} \subseteq \mathbb{R}^{n_{\nu}}$ the manifolds obtained from this corollary for $\widetilde{M}$. Since for each $\nu$ the projection $\Pi_{n} \mid M_{\nu}: M_{\nu} \longrightarrow \Pi_{n}\left(M_{\nu}\right) \subseteq \widetilde{M}$ is a diffeomorphism, it follows that for each $w \in M_{\nu}$ and $x=\Pi_{n}(w)$, we have $\operatorname{rk}\left(\Pi_{k}^{n_{\nu}} \mid T_{w} M_{\nu}\right) \leq \operatorname{rk}\left(\Pi_{k} \mid T_{x} M\right)<r(M)$, that is, $r\left(M_{\nu}\right)<r(M)$. Therefore, by the inductive hypothesis, $(*)$ holds with each $M_{\nu}$ in place of $M$, which together with Remark 4.3 finishes the proof of the lemma.

Proposition 4.7. Let $A \subseteq \mathbb{R}^{n}$ be a bounded $\mathcal{C}$-semianalytic set and $k \leq n$. Then there are trivial $\mathcal{C}$-semianalytic manifolds $N_{i} \subseteq \mathbb{R}^{n_{i}}$ with $n_{i} \geq n$ for $i=1, \ldots, J$, each $\Delta$-definable from $A$, such that

$$
\Pi_{k}(A)=\Pi_{k}\left(N_{1}\right) \cup \cdots \cup \Pi_{k}\left(N_{J}\right)
$$


and for each $i$ we have $d:=\operatorname{dim}\left(N_{i}\right) \leq k$, and there is a strictly increasing $\iota$ : $\{1, \ldots, d\} \longrightarrow\{1, \ldots, k\}$ such that $\Pi_{\iota} \mid N_{i}: N_{i} \longrightarrow \mathbb{R}^{d}$ is an immersion.

Proof. By induction on $e:=\operatorname{dim}(A)$ : if $e=0$, then $A$ is finite by Corollary 4.4, so the theorem is trivial in this case. So we assume $e>0$ and that the theorem holds for lower values of $e$.

Note first that if there is a bounded $\mathcal{C}$-semianalytic set $E \subseteq \mathbb{R}^{\tilde{n}}$ for some $\widetilde{n} \geq n$ such that $E$ is $\Delta$-definable from $A, A=\Pi_{n}(E)$ and the proposition holds with $E$ and $\widetilde{n}$ in place of $A$ and $n$, then by Remark 4.3 the proposition also holds for $A$ and $n$. Similarly, if $A$ is a finite union of $\mathcal{C}$-semianalytic sets each $\Delta$-definable from $A$ and satisfying the proposition in place of $A$, then again the proposition also holds for A. By Corollary 4.4 and the inductive hypothesis, reasoning as at the end of the previous proof and increasing $n$ if necessary, we may therefore reduce to the case that $A$ is a $\mathcal{C}$-manifold $M$ of dimension $e$.

Applying Lemma 4.6] to $M$ (with corresponding $n$ and $k$ as above), we let $N_{1}, \ldots, N_{K}$ and $A_{1}, \ldots, A_{L}$ be as in (*) for $M$. Since for each $j=1, \ldots, L$ we have $\operatorname{dim}\left(A_{j}\right)<e$, the inductive hypothesis and Remark 4.3 together with the above imply that we may even reduce to the case where $M=N_{i}$ for some $i \in\{1, \ldots, K\}$ (again increasing $n$ if necessary), that is, we may assume that there is a strictly increasing $\iota:\{1, \ldots, \operatorname{dim}(M)\} \longrightarrow\{1, \ldots, k\}$ such that $\Pi_{\iota} \mid M$ is an immersion.

Now we apply Corollary 4.4 again with $M$ in place of $A$, and we let $N$ (with corresponding $n^{\prime} \geq n$ ) be one of the $N_{i}$ 's thus obtained. Since $\Pi_{n} \mid N: N \longrightarrow$ $\Pi_{n}(N)$ is a diffeomorphism, $\Pi_{n}(N) \subseteq M$ and $\Pi_{\iota} \mid M$ is an immersion, we see that $\operatorname{dim}(N)=\operatorname{dim}(M) \leq k$ and $\Pi_{\iota}^{n^{\prime}} \mid N$ is an immersion.

\section{O-Minimality}

For $n \in \mathbb{N}$ we put, with $I=[-1,1]$,

$$
\Lambda_{n}:=\left\{X \subseteq I^{n}: X \text { is } \mathcal{C} \text {-semianalytic }\right\} .
$$

Then the system $\Lambda:=\left(\Lambda_{n}\right)_{n \in \mathbb{N}}$ satisfies axioms (I)-(III) of [8, Section 2].

Let $A \subseteq I^{n}$. In accordance with [8], we call $A$ a $\Lambda$-set if $A \in \Lambda_{n}$; if in addition $A$ is a manifold, we call $A$ a $\Lambda$-manifold. Similarly, $A$ is a sub- $\Lambda$-set if there are $m \geq n$ and $B \in \Lambda_{m}$ such that $A=\Pi_{n}(B)$; if in addition $A$ is a manifold, then $A$ is a sub- $\Lambda$-manifold.

We recall that $A \subseteq I^{n}$ has the $\Lambda$-Gabrielov property if for each $m \leq n$ there are connected sub- $\Lambda$-manifolds $B_{i} \subseteq I^{n+q_{i}}$, with $i=1, \ldots, k$ and $q_{1}, \ldots, q_{k} \geq 0$, such that

$$
\Pi_{m}(A)=\Pi_{m}\left(B_{1}\right) \cup \cdots \cup \Pi_{m}\left(B_{k}\right)
$$

and for each $i \in\{1, \ldots, k\}$ we have

(G1) fr $B_{i}$ is contained in a closed sub- $\Lambda$-set $D_{i} \subseteq I^{n+q_{i}}$ such that $D_{i}$ has dimension and $\operatorname{dim}\left(D_{i}\right)<\operatorname{dim}\left(B_{i}\right)$

(G2) $d:=\operatorname{dim}\left(B_{i}\right) \leq m$, and there is a strictly increasing $\lambda:\{1, \ldots, d\} \longrightarrow$ $\{1, \ldots, m\}$ such that $\Pi_{\lambda} \mid B_{i}: B_{i} \longrightarrow \mathbb{R}^{m}$ is an immersion.

From the previous section, we obtain

Corollary 5.1. Every $\Lambda$-set $A \subseteq I^{n}$ has the $\Lambda$-Gabrielov property. Moreover, the corresponding $B_{i}$ can be chosen to be $\Delta$-definable from $A$. 
Proof. Note first that if $A \subseteq I^{n}$ in Corollary 4.4 or in Proposition 4.7, then each $N_{i}$ can be taken to be a subset of $I^{n_{i}}$ (simply multiply the coordinates $x_{n+1}, \ldots, x_{n_{i}}$ by some small enough $\delta>0$ ). The corollary therefore follows from Proposition 4.7 and Remark 3.5.(1).

We now fix, for each $n \geq 1$, an arbitrary $\mathbb{R}$-subalgebra $\mathcal{D}_{n}$ of $\mathcal{C}_{n, 1}$ that is closed with respect to partial differentiation and contains the maps $x \mapsto x_{i}: I^{n} \longrightarrow I$ for $i=1, \ldots, n$. We let $\mathcal{F}=\bigcup_{n \in \mathbb{N}} \mathcal{D}_{n}$ and put $\mathbb{R}_{\mathcal{D}}:=\mathbb{R}(\mathcal{F})$.

In the case of Example 3.1(1), $\mathbb{R}_{\mathcal{C}}$ coincides with $\mathbb{R}_{\mathcal{C}(M)}$; thus Theorem 1 follows from the corresponding theorems below.

Theorem 5.2. The structure $\mathbb{R}_{\mathcal{D}}$ is model complete and o-minimal.

Proof. By well-known arguments (see [8, Corollary 2.8]), Corollary 5.1]implies that the structure $\left(I,\left\{A \subseteq I^{n}: A \in \Lambda_{n}\right.\right.$ and $\left.\left.n \in \mathbb{N}\right\}\right)$ is o-minimal. On the other hand, note that every $\Lambda$-set is quantifier-free definable in $\mathbb{R}_{\mathcal{C}}$, and for every quantifierfree definable set $A \subseteq \mathbb{R}^{n}$, the set $\tau_{n}(A)$ is a $\Lambda$-set, where $\tau_{n}\left(x_{1}, \ldots, x_{n}\right):=$ $\left(x_{1} / \sqrt{1+x_{1}^{2}}, \ldots, x_{n} / \sqrt{1+x_{n}^{2}}\right)$. Thus by [8, Corollary 2.9], $\mathbb{R}_{\mathcal{C}}$ is model complete and o-minimal. Finally, it follows from the proof of [8, Corollary 2.8] and the second statement of Corollary 5.1 that $\mathbb{R}_{\mathcal{D}}$ is also model complete.

Next, we show that $\mathbb{R}_{\mathcal{D}}$ is polynomially bounded. (Recall that an expansion $\mathcal{R}$ of the real field is polynomially bounded if for every definable function $f: \mathbb{R} \longrightarrow \mathbb{R}$ there is a $p \in \mathbb{N}$ such that $|f(t)| \leq t^{p}$ for all sufficiently large $t$.)

Lemma 5.3 (Curve Selection). Let $B \subseteq \mathbb{R}^{n}$ be definable in $\mathbb{R}_{\mathcal{C}}$, and let $0 \in \operatorname{bd}(B)$. Then there exists a $g=\left(g_{1}, \ldots, g_{n}\right) \in\left(\mathcal{C}_{1}\right)^{n}$ such that $g(0)=0$ and $g(t) \in B$ for all sufficiently small $t>0$.

Proof. We may clearly assume that $B$ is bounded. By Theorem 5.2 and because $\mathbb{R}_{\mathcal{C}}$ is an expansion of the real field, we may even assume that $B=\Pi_{n}(D)$ for some bounded $\mathcal{C}$-semianalytic set $D \subseteq \mathbb{R}^{m}$ with $m \geq n$. Thus the curve selection lemma follows from Corollary 4.4 and Remark 3.5 (2).

Theorem 5.4. $\mathbb{R}_{\mathcal{D}}$ is polynomially bounded, and the function $x^{\lambda}:(0,1) \longrightarrow \mathbb{R}$ is definable in $\mathbb{R}_{\mathcal{D}}$ if and only if $\lambda \in \mathbb{Q}$.

Proof. It clearly suffices to prove the theorem for $\mathbb{R}_{\mathcal{C}}$. Let $\epsilon>0$ and let $f:(0, \epsilon) \longrightarrow$ $\mathbb{R}$ be definable in $\mathbb{R}_{\mathcal{C}}$; we show that for some nonzero $c \in \mathbb{R}$ and $r \in \mathbb{Q}$ we have $\lim _{x \rightarrow 0^{+}} f(x) / x^{r}=c$.

Replacing $f$ by $1 / f$ if necessary, we may clearly assume that $\lim _{x \rightarrow 0} f(t)=0$. Then $(0,0) \in \operatorname{fr}(\Gamma(f))$, so by Lemma 5.3 there are $\delta>0$ and $g_{1}, g_{2} \in \mathcal{C}_{1}$ such that $\left(g_{1}(t), g_{2}(t)\right) \in \Gamma(f)$ for all $t \in(0, \delta)$ and $g_{1}(0)=g_{2}(0)=0$. Let $p, q \in$ $\mathbb{N} \backslash\{0\}, a, b \in \mathbb{R} \backslash\{0\}$ and $h_{1}, h_{2} \in \mathcal{C}_{1}$ be such that $g_{1}(T)=T^{p}\left(a+h_{1}(T)\right)$ and $g_{2}(T)=T^{q}\left(b+h_{2}(T)\right)$ and $h_{1}(0)=h_{2}(0)=0$. Since for all sufficiently small $x>0$ there is a $t \in(0, \delta)$ such that $(x, f(x))=\left(g_{1}(t), g_{2}(t)\right)$, it follows that $\lim _{x \rightarrow 0^{+}} f(x) / x^{q / p}=b / a^{q / p}$.

Remark. Let $\mathcal{C}_{B}^{\prime}$ be the system associated to $\mathcal{R}=\mathbb{R}_{\mathcal{C}}$ as in Example 3.1(2). Then for each $n$, we have $\mathcal{C}_{n} \subseteq \mathcal{C}_{n}^{\prime}$, but we do not know if $\mathcal{C}_{n}=\mathcal{C}_{n}^{\prime}$. The latter equality is related to the open question whether $\mathbb{R}_{\mathcal{C}}$ admits quantifier elimination or even a preparation theorem in the spirit of [17] or [13].

Finally, we show that $\mathbb{R}_{\mathcal{D}}$ admits $C^{\infty}$ cell decomposition. 
Lemma 5.5. Let $A \subseteq \mathbb{R}^{n}$ be definable in $\mathbb{R}_{\mathcal{D}}$. Then $A$ is a finite union of $C^{\infty}$ manifolds that are definable in $\mathbb{R}_{\mathcal{D}}$.

Proof. By induction on $d:=\operatorname{dim}(A)$; the case $d=0$ is trivial, so we assume that $d>0$ and the lemma holds for lower values of $d$. By model completeness, there is a $\mathcal{C}$-semianalytic set $C \subseteq \mathbb{R}^{n^{\prime}}$ with $n^{\prime} \geq n$ such that $A=\Pi_{n}(C)$ and $C$ is definable in $\mathbb{R}_{\mathcal{D}}$. Using an analytic, semialgebraic diffeomorphism mapping $\mathbb{R}^{n}$ onto $(-1,1)^{n}$, we may assume that $A$ and $C$ are bounded. Thus by Proposition 4.7, we may even assume that there is a $\mathcal{C}$-semianalytic manifold $B \subseteq \mathbb{R}^{m}$ with $n \leq m$, definable in $\mathbb{R}_{\mathcal{D}}$, such that $A=\Pi_{n}(B)$ and $\Pi_{n} \mid B$ is an immersion. Using cell decomposition, we now partition $B$ into cells $B_{1}, \ldots, B_{k}$ definable in $\mathbb{R}_{\mathcal{D}}$. By the inductive hypothesis, it suffices to show that if $i \in\{1, \ldots, k\}$ is such that $\operatorname{dim}\left(\Pi_{n} B_{i}\right)=d$, then $\Pi_{n}\left(B_{i}\right)$ is a $C^{\infty}$ manifold. Fix such an $i$; since $\Pi_{n} \mid B$ is an immersion, we have $\operatorname{dim}(B)=d$, so that $\operatorname{dim}\left(B_{i}\right)=\operatorname{dim}\left(\Pi_{n} B_{i}\right)$. Since $B_{i}$ is a cell, it follows that $\Pi_{n} \mid B_{i}$ is a homeomorphism onto $\Pi_{n}\left(B_{i}\right)$. Also, $B_{i}$ must be open in $B$, and hence is itself a $C^{\infty}$ manifold. Thus the image $\Pi_{n}\left(B_{i}\right)$ of the $C^{\infty}$ immersion $\Pi_{n} \mid B_{i}$ is a $C^{\infty}$ manifold.

Theorem 5.6. $\mathbb{R}_{\mathcal{D}}$ admits $C^{\infty}$ cell decomposition.

Proof. We show by induction on $n$ that if $\mathcal{A}$ is a finite collection of subsets of $\mathbb{R}^{n}$ definable in $\mathbb{R}_{\mathcal{D}}$, then there is a decomposition of $\mathbb{R}^{n}$ into $C^{\infty}$ cells definable in $\mathbb{R}_{\mathcal{D}}$ that is compatible with each member of $\mathcal{A}$. The cases $n=0,1$ are trivial, so we assume that $n>1$. Let $f: A \longrightarrow \mathbb{R}$ be a $C^{1}$ function definable in $\mathbb{R}_{\mathcal{D}}$ with $A \subseteq \mathbb{R}^{n-1}$ a $C^{1}$ cell. It suffices to partition $A$ into $C^{\infty}$ cells $A_{1}, \ldots, A_{K}$ definable in $\mathbb{R}_{\mathcal{D}}$ such that $f \mid A_{j}$ is $C^{\infty}$ for each $j$. This follows from the inductive hypothesis by applying Lemma 5.5 to $\Gamma(f)$.

\section{Appendix: Denjoy-Carleman Classes and the sum theorem}

Let $M=\left(M_{0}, M_{1}, \ldots\right)$ with $1 \leq M_{0} \leq M_{1} \leq \ldots$ be a sequence of real numbers. We assume that $M$ is strongly log-convex, that is,

$$
\forall k \geq 1, \quad\left(\frac{M_{k}}{k !}\right)^{2} \leq \frac{M_{k+1}}{(k+1) !} \cdot \frac{M_{k-1}}{(k-1) !} .
$$

Recall that $M^{(j)}=\left(M_{i+j}\right)_{i}$ for each $j \in \mathbb{N}$. The following elementary observations were pointed out to us by $\mathrm{V}$. Thilliez:

Lemma 6.1. (1) Let $m_{0}:=M_{0}$ and $m_{i}:=\left(M_{i} / i !\right)^{1 /(i-1)}$ for $i \geq 1$. Then the sequence $m=\left(m_{0}, m_{1}, \ldots\right)$ is almost increasing, that is, there is a constant $C \geq 1$ such that $m_{i} \leq C \cdot m_{j}$ for all $j \geq i \geq 2$.

(2) Let $N_{i}^{(j)}:=\frac{i !}{(i+j) !} M_{i+j}$ for each $i, j \in \mathbb{N}$. Then $N^{(j)}:=\left(N_{i}^{(j)}\right)$ is strongly log-convex and $\mathcal{C}_{B}^{0}\left(M^{(j)}\right)=\mathcal{C}_{B}^{0}\left(N^{(j)}\right)$ for each $j$ and every compact box $B$.

Proof. (1) Note first that $m$ is almost increasing if and only if the sequence $\widetilde{m}=$ $\left(\widetilde{m}_{0}, \widetilde{m}_{1}, \ldots\right)$ is almost increasing, where $\widetilde{m}_{0}:=1$ and $\widetilde{m}_{i}:=\left(\frac{M_{i} / M_{0}}{i !}\right)^{1 /(i-1)}$ for $i \geq 1$. We may therefore assume that $m_{0}=M_{0}=1$.

We put $l_{i}:=\log \left(M_{i} / i\right.$ !) for $i \geq 1$. A straightforward induction on $i$ now proves $i \cdot l_{i-1} \leq(i-1) l_{i}$ for all $i \geq 1$. Applying this repeatedly, we get for $j \geq i \geq 2$ :

$$
(j-1) l_{i} \leq(j-1) \frac{i}{i+1} l_{i+1} \leq \cdots \leq(j-1) \frac{i}{j} l_{j} \leq 2(i-1) l_{j} .
$$


(2) It is straightforward to see that $N^{(j)}$ satisfies SLC for each $j$. Moreover, $\mathcal{C}_{B}^{0}\left(M^{(j)}\right)=\mathcal{C}_{B}^{0}\left(N^{(j)}\right)$ follows from the fact that $j ! i ! \leq(i+j) ! \leq\left(2^{j}\right)^{i} j ! i$ ! for all $i, j \in \mathbb{N}$.

The following is a noteworthy consequence of the previous lemma:

Corollary 6.2. For every compact box $B \subseteq \mathbb{R}^{n}, n \in \mathbb{N}$, the class $\mathcal{C}_{B}^{0}(M)$ contains all functions that are analytic on a neighborhood of $B$.

Proof. The proof of Lemma 6.11) shows in particular that the sequence of all $\left(\frac{M_{i} / M_{0}}{i !}\right)^{1 / i}$ is nondecreasing. It follows (by induction on $i$ ) that $\frac{M_{i} / M_{0}}{i !} \geq 1$ for all $i$, that is, $M_{i} \geq M_{0} i$ !.

Together with 12 and 18, Lemma 6.1 also implies

Proposition 6.3. (1) Let $d, e \geq 1, f \in \mathcal{C}_{[-1,1]^{d}}^{0}(M)$ and $g_{i} \in \mathcal{C}_{[-1,1]^{e}}^{0}(M)$ for $i=1, \ldots, d$. Suppose that $g_{i}\left([-1,1]^{e}\right) \subseteq[-1,1]$ for each $i$ and put $g:=\left(g_{1}, \ldots, g_{d}\right)$. Then $f \circ g \in \mathcal{C}_{[-1,1]}^{0}(M)$.

(2) Let $f \in \mathcal{C}_{[-1,1]^{d+1}}^{0}(M)$, and let $g:[-1,1]^{d} \longrightarrow[-1,1]$ be a $C^{\infty}$ function such that $f\left(x_{1}, \ldots, x_{d}, g\left(x_{1}, \ldots, x_{d}\right)\right)=0$ and $\frac{\partial f}{\partial x_{d+1}}\left(x_{1}, \ldots, x_{d+1}\right) \neq 0$ for all $x_{1}, \ldots, x_{d+1} \in[-1,1]$. Then $g$ belongs to $\mathcal{C}_{[-1,1]^{d}}^{0}(M)$.

We now obtain the following from Proposition 6.3 and Lemma 6.1 (2):

Corollary 6.4. If $M$ is strongly log-convex, then the system of all $\mathcal{C}_{B}(M)$, with $B \subseteq \mathbb{R}^{n}$ a compact box and $n \in \mathbb{N}$, is closed under composition and extracting implicit functions.

Using the previous corollary, an elementary argument shows that if $B \subseteq \mathbb{R}^{n}$ is a compact box and $0 \in B$, and if $f \in \mathcal{C}_{B}(M)$ and $i \in\{1, \ldots, n\}$ are such that $f\left(x_{1}, \ldots, x_{i-1}, 0, x_{i+1}, \ldots, x_{n}\right)=0$ for all sufficiently small $x_{1}, \ldots, x_{i-1}, x_{i+1}, \ldots$, $x_{n} \in \mathbb{R}$, there exist a compact box $B^{\prime} \subseteq B$ containing 0 and $g \in \mathcal{C}_{B^{\prime}}(M)$ such that $f(x)=x_{i} \cdot g(x)$ for all $x \in B^{\prime}$. Hence

Corollary 6.5. If $M$ is residually log-convex, then the system of all $\mathcal{C}_{B}(M)$, with $B \subseteq \mathbb{R}^{n}$ a compact box and $n \in \mathbb{N}$, satisfies (C1)-(C7).

Finally, we aim to prove Theorem 2 ; it will follow, via elementary facts on Fourier series, from the following result.

Lemma 6.6. Suppose that $\sum_{n=0}^{\infty} \gamma_{n}$ is a series of nonnegative real numbers with the property that, for each $k \geq 0$, the series $\sum_{n=0}^{\infty} n^{k} \gamma_{n}$ converges. Then there exist strongly log-convex sequences $M=\left(M_{0}, M_{1}, \ldots\right)$ and $N=\left(N_{0}, N_{1}, \ldots\right)$, both satisfying QA, and a subset $S \subseteq \mathbb{N}$, such that, for all $k \geq 0$,

$$
\sum_{n \in S} n^{k} \gamma_{n} \leq M_{k} \quad \text { and } \quad \sum_{n \in \mathbb{N} \backslash S} n^{k} \gamma_{n} \leq N_{k}
$$

Proof. (Following [11 p. 117]) We introduce constants $c_{0}:=\max \left\{1, \sum_{n=0}^{\infty} \gamma_{n}\right\}$ and $c_{1}:=\max \left\{c_{0}, \sum_{n=0}^{\infty} n \gamma_{n}\right\}$ and define $M_{0}=N_{0}:=c_{0}$ and $M_{1}=N_{1}:=c_{1}$. Let $\lambda_{0}:=1$ and suppose, for an inductive construction, that we have defined 
integers $\lambda_{0}<\mu_{1}<\lambda_{1}<\mu_{2}<\cdots<\mu_{j}<\lambda_{j}$ (for some $j \geq 0$ ) and real numbers $M_{0} \leq M_{1} \leq \cdots \leq M_{\lambda_{j}}, N_{0} \leq N_{1} \leq \cdots \leq N_{\lambda_{j}}$, satisfying the following conditions:

$$
\begin{aligned}
& \sum_{k=1+\lambda_{i}}^{\mu_{i+1}} \frac{M_{k-1}}{M_{k}} \geq 1, \quad \text { for each even } i \text { with } 0 \leq i<j ; \\
& \sum_{k=1+\lambda_{i}}^{\mu_{i+1}} \frac{N_{k-1}}{N_{k}} \geq 1, \quad \text { for each odd } i \text { with } 0<i<j ;
\end{aligned}
$$

and for all $i$ with $0 \leq i<\lambda_{j}$

$$
\begin{aligned}
& \left(\frac{M_{i}}{i !}\right)^{2} \leq\left(\frac{M_{i+1}}{(i+1) !}\right)\left(\frac{M_{i-1}}{(i-1) !}\right) \text { and } \\
& \left(\frac{N_{i}}{i !}\right)^{2} \leq\left(\frac{N_{i+1}}{(i+1) !}\right)\left(\frac{N_{i-1}}{(i-1) !}\right) .
\end{aligned}
$$

We extend these sequences as follows:

Case 1. $j$ is even. Choose $\mu_{j+1}>\lambda_{j}$ large enough so that

$$
\sum_{k=1+\lambda_{j}}^{\mu_{j+1}} \frac{1}{k} \geq \max \left\{M_{\lambda_{j}}, c_{0}\left(1+\lambda_{j}\right)^{1+\lambda_{j}}\right\} .
$$

Now choose $\lambda_{j+1}>\mu_{j+1}$ large enough so that $\sum_{n=1+\lambda_{j+1}}^{\infty} n^{\mu_{j+1}} \gamma_{n}<1$. Now set

$$
M_{k}:= \begin{cases}\max \left\{\frac{k M_{k-1}^{2}}{(k-1) M_{k-2}}, c_{0}\left(1+\lambda_{j}\right)^{k}\right\} & \text { for } \lambda_{j}<k \leq \mu_{j+1}, \\ \max \left\{\frac{k M_{k-1}^{2}}{(k-1) M_{k-2}}, \sum_{n=0}^{\infty} n^{k} \gamma_{n}\right\} & \text { for } \mu_{j+1}<k \leq \lambda_{j+1}\end{cases}
$$

and

$$
N_{k}:=\max \left\{\frac{k N_{k-1}^{2}}{(k-1) N_{k-2}}, \sum_{n=0}^{\infty} n^{k} \gamma_{n}\right\} \quad \text { for } \lambda_{j}<k \leq \lambda_{j+1} .
$$

(Notice that $k-1>0$ for the values of $k, j$ considered above.) It follows immediately by induction that the $M_{k}$ 's and $N_{k}$ 's are increasing and $\geq 1$.

We claim that $M_{k} / M_{k-1} \leq k \theta$, for all $k$ with $\lambda_{j}<k \leq \mu_{j+1}$, where $\theta:=$ $\max \left\{M_{\lambda_{j}}, c_{0}\left(1+\lambda_{j}\right)^{1+\lambda_{j}}\right\}$. For this is clear if $k=1+\lambda_{j}$ by definition of $M_{1+\lambda_{j}}$. Also, if $1+\lambda_{j}<k \leq \mu_{j+1}$, then either

$$
\begin{aligned}
\frac{M_{k}}{M_{k-1}} & =\frac{k}{k-1} \frac{M_{k-1}}{M_{k-2}} \\
& \leq \frac{k}{k-1} \cdot(k-1) \theta \quad \text { (by inductive hypothesis) } \\
& =k \theta
\end{aligned}
$$

or

$$
\frac{M_{k}}{M_{k-1}}=\frac{c_{0}\left(1+\lambda_{j}\right)^{k}}{M_{k-1}} \leq \frac{c_{0}\left(1+\lambda_{j}\right)^{k}}{c_{0}\left(1+\lambda_{j}\right)^{k-1}} \leq 1+\lambda_{j} \leq \theta \leq k \theta .
$$

This completes the proof of the claim, from which it follows that

$$
\sum_{k=1+\lambda_{j}}^{\mu_{j+1}} \frac{M_{k-1}}{M_{k}} \geq \sum_{k=1+\lambda_{j}}^{\mu_{j+1}} \frac{1}{k \theta} \geq 1 \quad \text { (by definition of } \mu_{j+1} \text { ). }
$$


This and condition $(1)_{j}$ imply condition $(1)_{j+1}$. Clearly condition $(2)_{j}$ implies condition $(2)_{j+1}$, since $j$ is even. Conditions $(3)_{j+1}$ and $(4)_{j+1}$ follow directly from the definitions of $M_{k}$ and $N_{k}$ for $\lambda_{j}<k \leq \lambda_{j+1}$.

Case 2. $j$ is odd. The construction here is the same as in the even case, except that the roles of the $M_{k}$ 's and $N_{k}$ 's are interchanged.

This completes the construction of the sequences $M=\left(M_{0}, M_{1}, \ldots\right)$ and $N=$ $\left(N_{0}, N_{1}, \ldots\right)$. They both satisfy (SLC) by conditions $(3)_{j},(4)_{j}$ and (QA) by conditions $(1)_{j},(2)_{j}($ for $j=0,1, \ldots)$.

Now set $S:=\left\{k \in \mathbb{N}: \lambda_{j}<k \leq \lambda_{j+1}\right.$ for some odd $\left.j \in \mathbb{N}\right\}$. To complete the proof of the lemma, let $k \geq 0$ be given. If $k=0$ or $k=1$, then certainly $\sum_{n \in S} n^{k} \gamma_{n} \leq \sum_{n=0}^{\infty} n^{k} \gamma_{n} \leq M_{k}$ (by definition of $M_{0}, M_{1}$ ) and $\sum_{n \in \mathbb{N} \backslash S} n^{k} \gamma_{n} \leq$ $\sum_{n=0}^{\infty} n^{k} \gamma_{n} \leq N_{k}$ (by definition of $N_{0}, N_{1}$ ). So we assume $k>1$ and let $j$ be the unique $i>0$ such that $\lambda_{i}<k \leq \lambda_{i+1}$.

If $j$ is even, then $\sum_{n \in \mathbb{N} \backslash S} n^{k} \gamma_{n} \leq \sum_{n=0}^{\infty} n^{k} \gamma_{n} \leq N_{k}$ (by definition of $N_{k}$ ). Similarly, if $\mu_{j+1}<k \leq \lambda_{j+1}$, then $\sum_{n \in S} n^{k} \gamma_{n} \leq \sum_{n=0}^{\infty} n^{k} \gamma_{n} \leq M_{k}$ (by definition of $M_{k}$ ). Finally, if $\lambda_{j}<k \leq \mu_{j+1}$, then

$$
\sum_{n \in S} n^{k} \gamma_{n} \leq \sum_{n \leq \lambda_{j}} n^{k} \gamma_{n}+\sum_{n=1+\lambda_{j+1}}^{\infty} n^{k} \gamma_{n}
$$

(by definition of $S$, since $j$ is even). Thus

$$
\begin{aligned}
\sum_{n \in S} n^{k} \gamma_{n} & \leq \lambda_{j}^{k} \cdot \sum_{n=0}^{\infty} \gamma_{n}+\sum_{n=1+\lambda_{j+1}}^{\infty} n^{\mu_{j+1}} \gamma_{n} \\
& \leq c_{0} \lambda_{j}^{k}+1 \quad\left(\text { by definition of } \lambda_{j+1} \text { and of } c_{0}\right) \\
& \leq c_{0}\left(1+\lambda_{j}\right)^{k} \quad\left(\text { as } c_{0} \geq 1\right) \\
& \leq M_{k} \quad\left(\text { by the definition of } M_{k} \text { for } \lambda_{j}<k \leq \mu_{j+1}\right) .
\end{aligned}
$$

If $j$ is odd, the proof is similar with the roles of $M_{k}$ and $N_{k}$ interchanged. This completes the proof of the lemma.

Now suppose that $f:[-1,1]^{d} \longrightarrow \mathbb{R}$ is a $C^{\infty}$ function (for some $d \geq 1$ ). Extend $f$ to a $C^{\infty}$ function $f^{*}: \mathbb{R}^{d} \longrightarrow \mathbb{R}$. Define $g: \mathbb{R}^{d} \longrightarrow \mathbb{R}$ by $g\left(\theta_{1}, \ldots, \theta_{d}\right):=$ $f^{*}\left(3 \cos \theta_{1}, \ldots, 3 \cos \theta_{d}\right)$. Then $g$ is a $C^{\infty}$ function which is $2 \pi$-periodic and even (in each variable). The Fourier series of $g$ may be therefore written in the form

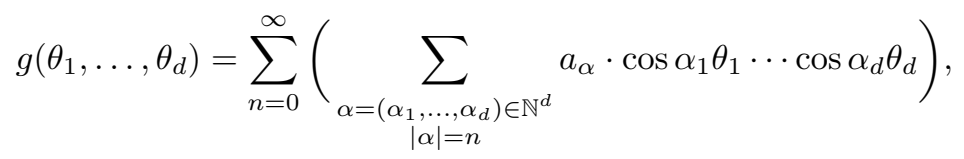

where the Fourier coefficients $a_{\alpha}$ are given by

$$
a_{\alpha}=\frac{1}{(2 \pi)^{d}} \int_{-\pi}^{\pi} \cdots \int_{-\pi}^{\pi} g\left(\theta_{1}, \ldots, \theta_{d}\right) \cdot \cos \alpha_{1} \theta_{1} \cdots \cos \alpha_{d} \theta_{d} \cdot d \theta_{1} \cdots d \theta_{d} .
$$

Now, for each $k \geq 0$, let

$$
A_{k}:=\sup \left\{\left|g^{(\beta)}\left(\theta_{1}, \ldots, \theta_{d}\right)\right|: \beta \in \mathbb{N}^{d},|\beta|=k, \theta_{1}, \ldots, \theta_{d} \in \mathbb{R}\right\} .
$$


Suppose that $i$ is such that $1 \leq i \leq d$ and $\alpha_{i} \neq 0$ in (6.2). Then repeated integration by parts of (6.2) (with respect to the variable $\theta_{i}$ ) yields, for any $k \geq 0,\left|a_{\alpha}\right| \leq$ $A_{0}^{d-1} \cdot A_{k} \cdot \alpha_{i}^{-k}$. Now if $n \geq 1$ and $\alpha \in \mathbb{N}^{d}$ satisfies $|\alpha|=n$, then we may choose $i$ such that $\alpha_{i} \geq n / d$, and hence it follows that $\left|a_{\alpha}\right| \leq A_{0}^{d-1} \cdot A_{k} \cdot d^{k} \cdot n^{-k}$ for all $k \geq 0$. Thus

$$
\sum_{\substack{\alpha \in \mathbb{N}^{d} \\|\alpha|=n}}\left|a_{\alpha}\right| \leq(n+1)^{d} \cdot A_{0}^{d} \cdot A_{k} \cdot d^{k} \cdot n^{-k} \quad \text { for all } k \geq 0 .
$$

In particular, it follows that for $n \geq \max \left\{2, A_{0}\right\}$,

$$
\sum_{\substack{\alpha \in \mathbb{N}^{d} \\|\alpha|=n}}\left|a_{\alpha}\right| \leq A_{k+3 d+2} \cdot n^{-k-2} \quad \text { for all } k \geq 0 .
$$

So, if we set $\gamma_{n}:=\sum_{\substack{\alpha \in \mathbb{N}^{d} \\|\alpha|=n}}\left|a_{\alpha}\right|$ (for $n \geq 0$ ), then (6.3) implies that $\sum_{n=0}^{\infty} n^{k} \gamma_{n}$ converges for all $k \geq 0$, and we may apply the lemma to obtain strongly log-convex sequences $M$ and $N$, both satisfying (QA), and a subset $S \subseteq \mathbb{N}$ satisfying the conclusion of the lemma. Define

$$
g_{i}\left(\theta_{1}, \ldots, \theta_{d}\right):=\sum_{n \in S_{i}}\left(\sum_{\begin{array}{c}
\alpha=\left(\alpha_{1}, \ldots, \alpha_{d}\right) \in \mathbb{N}^{d} \\
|\alpha|=n
\end{array}} a_{\alpha} \cdot \cos \alpha_{1} \theta_{1} \cdots \cos \alpha_{d} \theta_{d}\right),
$$

for $i=1,2$, where $S_{1}:=S$ and $S_{2}:=\mathbb{N} \backslash S$.

Clearly $g_{1}, g_{2}$ are $C^{\infty}$ functions and $g=g_{1}+g_{2}$. Furthermore, (6.3) readily implies that the series in (6.4) may be differentiated term by term any number of times (to yield the corresponding derivatives of $g_{1}, g_{2}$ ), so we obtain, for any $k \geq 0$ and $\beta=\left(\beta_{1}, \ldots, \beta_{d}\right) \in \mathbb{N}^{d}$ with $|\beta|=k$,

$$
\begin{aligned}
\left|g_{i}^{(\beta)}\left(\theta_{1}, \ldots, \theta_{d}\right)\right| & \leq \sum_{n \in S_{i}}\left(\sum_{\substack{\alpha=\left(\alpha_{1}, \ldots, \alpha_{d}\right) \in \mathbb{N}^{d} \\
|\alpha|=n}}\left|a_{\alpha}\right| \cdot \alpha_{1}^{\beta_{1}} \cdots \alpha_{d}^{\beta_{d}}\right) \\
& \leq \sum_{n \in S_{i}} n^{k} \sum_{\begin{array}{c}
\alpha \in \mathbb{N}^{d} \\
|\alpha|=n
\end{array}}\left|a_{\alpha}\right|=\sum_{n \in S_{i}} n^{k} \gamma_{n} \\
& \leq \begin{cases}M_{k} & \text { if } i=1, \\
N_{k} & \text { if } i=2 .\end{cases}
\end{aligned}
$$

Now choose an inverse of the function $3 \cdot \cos \theta, \cos ^{-1} \frac{x}{3}$ say, which is analytic on the interval $(-2,2)$. By Corollary 6.2 , the restriction of $\cos ^{-1} \frac{x}{3}$ to $[-1,1]$ belongs to $\mathcal{C}_{[-1,1]}^{0}(M)$ and to $\mathcal{C}_{[-1,1]}^{0}(N)$. Thus, by setting

$$
f_{i}\left(x_{1}, \ldots, x_{d}\right):=g_{i}\left(\cos ^{-1} \frac{x_{1}}{3}, \ldots, \cos ^{-1} \frac{x_{d}}{3}\right)
$$

for $i=1,2$ and $x \in[-1,1]^{d}$, we have established part (1) of Theorem 2 .

Now let $f:[-1,1] \longrightarrow \mathbb{R}$ be a $C^{\infty}$ function whose Taylor series at every $x \in$ $[-1,1]$ is divergent, and apply part (1) of Theorem 2 to $f$. By quasianalyticity and Corollary 6.2, one of the two summands $f_{1}, f_{2}$ thus obtained must have a divergent Taylor series at every $x$ belonging to some open interval $I \subseteq[-1,1]$. Therefore, part (2) of Theorem 2 follows from part (1). 
In fact, Lemma 6.6 implies rather more, for an easy diagonalization shows that any countable sequence of series satisfying the hypotheses of the lemma can be majorized by a series that also satisfies these hypotheses. Hence

Theorem 6.7. Let $\mathcal{S}$ be a countable collection of $C^{\infty}$ real valued functions, each defined on an open neighourhood of $[-1,1]^{d}$ for some $d \geq 1$. Then there exist strongly log-convex sequences $M$ and $N$, both satisfying (QA), such that for each $f \in \mathcal{S}$ with $[-1,1]^{d}$ contained in the domain of $f$, there are $f_{1} \in \mathcal{C}_{[-1,1]^{d}}^{0}(M)$ and $f_{2} \in \mathcal{C}_{[-1,1]^{d}}^{0}(N)$ such that $f=f_{1}+f_{2}$.

\section{ADDED AFTER POSTING}

Dan Miller pointed out an error concerning Theorem [5.2; the assumptions on the systems $\mathcal{D}$, as stated before Theorem 5.2 are too weak. Indeed, they do not guarantee the following property needed for our arguments: if $A \subseteq \mathbb{R}^{n}$ is quantifierfree definable in $\mathcal{R}_{\mathcal{D}}$, then for every $a \in \mathbb{R}^{n}$ there is an $r \in(0, \infty)^{n}$ such that $(A-a) \cap I_{r}$ is a finite union of sets of the form

$$
\left\{x \in I_{r}: f(x / r)=0, g_{1}(x / r)>0, \ldots, g_{k}(x / r)>0\right\},
$$

where $f, g_{1}, \ldots, g_{k} \in \mathcal{D}_{n}$ and $x / r:=\left(x_{1} / r_{1}, \ldots, x_{n} / r_{n}\right)$. (More specifically, we need this property in Corollary 4.4 to guarantee $\Delta$-definability as stated there.)

Therefore, Theorem 5.2 only applies to systems $\mathcal{D}=\left(\mathcal{D}_{n}\right)$ such that for each $n \geq 1, \mathcal{D}_{n}$ is an $\mathbb{R}$-subalgebra of $\mathcal{C}_{n, 1}$ satisfying

(D1) $\mathcal{D}_{n}$ is closed with respect to partial differentiation and contains the maps $x \mapsto x_{i}: I^{n} \longrightarrow I$ for $i=1, \ldots, n$, and

(D2) for every $f \in \mathcal{D}_{n}$ there are an $\epsilon>1$ and a $g \in \mathcal{C}_{n, \epsilon}$ such that the function $x \mapsto g(\epsilon x): I^{n} \longrightarrow \mathbb{R}$ belongs to $\mathcal{D}_{n}$.

Of course, if $\mathcal{D}=\mathcal{C}$, then $\mathcal{D}$ satisfies (D1) and (D2) by hypothesis. On the other hand, the extra assumption (D2) makes our proof less "explicit" than that in [10].

\section{REFERENCES}

[1] S. S. Abhyankar and T. T. Moh, Newton-Puiseux expansion and generalized Tschirnhausen transformation I, J. Reine Angew. Math., 260 (1973), pp. 47-83. MR 49:2724

[2] - Newton-Puiseux expansion and generalized Tschirnhausen transformation II, J. Reine Angew. Math., 261 (1973), pp. 29-54. MR 49:2724

[3] E. Bierstone and P. Milman, Semianalytic and subanalytic sets, Inst. Hautes Études Sci. Publ. Math., 67 (1988), pp. 5-42. MR 89k:32011

[4] Canonical desingularization in characteristic zero by blowing up the maximum strata of a local invariant, Invent. Math., 128 (1997), pp. 207-302. MR 98e:14010

[5] C. L. Childress, Weierstrass division in quasianalytic local rings, Canad. J. Math., 28 (1976), pp. 938-953. MR 54:5491

[6] L. van den Dries, O-minimal structures and real analytic geometry, in Current developments in mathematics, 1998, International Press, 1999, pp. 105-152. MR 2001j:03075

[7] L. van den Dries and C. Miller, Geometric categories and o-minimal structures, Duke Math. J., 84 (1996), pp. 497-540. MR 97i:32008

[8] L. van den Dries and P. Speissegger, The real field with convergent generalized power series is model complete and o-minimal, Trans. Amer. Math. Soc., 350 (1998), pp. 4377-4421. MR 99a:03036

[9] ㄴ The field of reals with multisummable series and the exponential function, Proc. London Math. Soc. (3), 81 (2000), pp. 513-565. MR 2002k:03057

[10] A. Gabrielov, Complements of subanalytic sets and existential formulas for analytic functions, Invent. Math., 125 (1996), pp. 1-12. MR 97h:32007

[11] Y. Katznelson, Introduction to Harmonic Analysis, John Wiley, 1968. MR 40:1734 
[12] H. Komatsu, The implicit function theorem for ultradifferentiable mappings, Proc. Japan Acad., Ser. A, 55 (1979), pp. 69-72. MR 80e:58007

[13] J.-M. Lion and J.-P. Rolin, Théorème de préparation pour les fonctions logarithmicoexponentielles, Ann. Inst. Fourier, 47 (1997), pp. 859-884. MR 98h:32009

[14] S. Mandelbrojt, Sur les fonctions indéfiniment dérivables, Acta Math., 72 (1940), pp. 15-29. MR 1:297d

[15] C. Miller, Expansions of the real field with power functions, Ann. Pure Appl. Logic, 68 (1994), pp. 79-94. MR 95i:03081

[16] _ Infinite differentiability in polynomially bounded o-minimal structures, Proc. Amer. Math. Soc., 123 (1995), pp. 2551-2555. MR 95j:03069

[17] A. Parusiński, Lipschitz stratification of subanalytic sets, Ann. Sci. Ecole Norm. Sup. (4), 27 (1994), pp. 661-996. MR 96g:32017

[18] C. Roumieu, Ultradistributions définies sur $\mathbb{R} n$ et sur certaines classes de variétés différentiables, J. Anal. Math., 10 (1962-1963), pp. 153-192. MR 28:1487]

[19] W. Rudin, Real and Complex Analysis, McGraw-Hill, 1987. MR 88k:00002

Laboratoire de Topologie, Université de Bourgogne, 9 Av. Alain Savary, B.P. 47870, 21078 Dijon Cedex, France

E-mail address: rolin@u-bourgogne.fr

Department of Mathematics, University of Wisconsin, 480 Lincoln Drive, Madison, WISCONSIN 53706

E-mail address: speisseg@math.wisc.edu

Mathematical Institute, University of Oxford, 24-29 St. Giles', Oxford OX1 3LB, UNITED Kingdom

E-mail address: wilkie@maths.ox.ac.uk 